\title{
A TWO-PHASE SCENARIO FOR BULGE ASSEMBLY IN $\Lambda$ CDM COSMOLOGIES
}

\author{
A. Obreja ${ }^{1}$, R. Domínguez-Tenreiro ${ }^{1}$, C. Brook $^{1}$, F. J. Martínez-Serrano ${ }^{2}$, \\ M. Doménech-Moral ${ }^{2}$, A. Serna ${ }^{2}$, M. Mollá ${ }^{3}$, and G. STINSON ${ }^{4}$ \\ ${ }^{1}$ Departamento de Física Teórica, Universidad Autónoma de Madrid, E-28049 Cantoblanco Madrid, Spain; aura.obreja@uam.es \\ ${ }^{2}$ Departamento de Física y Arquitectura de Computadores, Universidad Miguel Hernández, E-03202 Elche, Spain \\ ${ }^{3}$ Departamento de Investigación Básica, CIEMAT, E-28040 Madrid, Spain \\ ${ }^{4}$ Max-Planck-Institut für Astronomie, Königstuhl 17, D-69117, Heidelberg, Germany \\ Received 2012 July 23; accepted 2012 November 11; published 2012 December 28
}

\begin{abstract}
We analyze and compare the bulges of a sample of $L_{*}$ spiral galaxies in hydrodynamical simulations in a cosmological context, using two different codes, P-DEVA and GASOLINE. The codes regulate star formation in very different ways, with P-DEVA simulations inputting low star formation efficiency under the assumption that feedback occurs on subgrid scales, while the GASOLINE simulations have feedback that drives large-scale outflows. In all cases, the marked knee shape in mass aggregation tracks, corresponding to the transition from an early phase of rapid mass assembly to a later slower one, separates the properties of two populations within the simulated bulges. The bulges analyzed show an important early starburst resulting from the collapse-like fast phase of mass assembly, followed by a second phase with lower star formation, driven by a variety of processes such as disk instabilities and/or mergers. Classifying bulge stellar particles identified at $z=0$ into old and young according to these two phases, we found bulge stellar sub-populations with distinct kinematics, shapes, stellar ages, and metal contents. The young components are more oblate, generally smaller, more rotationally supported, with higher metallicity and less alpha-element enhanced than the old ones. These results are consistent with the current observational status of bulges, and provide an explanation for some apparently paradoxical observations, such as bulge rejuvenation and metal-content gradients observed. Our results suggest that bulges of $L_{*}$ galaxies will generically have two bulge populations that can be likened to classical and pseudo-bulges, with differences being in the relative proportions of the two, which may vary due to galaxy mass and specific mass accretion and merger histories.
\end{abstract}

Key words: cosmology: theory -galaxies: bulges - galaxies: formation - galaxies: kinematics and dynamics galaxies: star formation - methods: numerical

Online-only material: color figures

\section{INTRODUCTION}

Understanding how bulges form and evolve is important within the theories and models of galaxy formation and evolution. Bulges are the component responsible for the central light in excess of the exponential disk (Freeman 1970), and account for more than $25 \%$ of the starlight emitted in the local universe. There are three main classes of observed bulge: classical, pseudo-, and boxy or peanut-shaped bulges; see Kormendy \& Kennicutt (2004) and Athanassoula (2005) for a detailed discussion on bulge classification. The distinction is important in that different classes cloud have formed and evolved in different ways.

The Milky Way Bulge is the only case in which individual stars are resolved, and thus provides unique information about bulge properties. The Milky Way is considered to have a boxy bulge, yet increased evidence for an old, $\alpha$-enriched stellar population formed on a short timescale has resulted in a twocomponent model (e.g., Tsujimoto \& Bekki 2012) of the bulge. It had been shown that two stellar populations coexist in the bulge, separated in age and metallicity (McWilliam \& Rich 1994; Feltzing \& Gilmore 2000; Barbuy et al. 1999; van Loon et al. 2003; Groenewegen \& Blommaert 2005; Zoccali et al. 2006; Fulbright et al. 2007; Zoccali et al. 2008) and that the separation somewhat extends to kinematics (Zhao et al. 1994; Soto et al. 2007), even if age determinations through color-magnitude diagrams showed that most bulge stars in the Galaxy are older than 10 Gyr (Ortolani et al. 1995; Feltzing \& Gilmore 2000; Zoccali et al. 2006; Clarkson et al. 2008).
Recently, Bensby et al. (2011) reported a 2-13 Gyr age span among microlensed turn-off bulge stars. These latest data from high-resolution spectrography have put increased emphasis on the picture of a two-component bulge. Indeed, analyses of giant stars confirmed the presence of two distinct populations: a metal-poor enriched in $[\alpha / \mathrm{Fe}]$ one with kinematics consistent with an old spheroid, and a metal-rich one with roughly solar $[\alpha / \mathrm{Fe}]$ and with bar-like kinematics (Babusiaux et al. 2010; Hill et al. 2011; Gonzalez et al. 2011; De Propris et al. 2011; Johnson et al. 2011; Robin et al. 2012; Soto et al. 2012; Ness \& Freeman 2012; Ness et al. 2012). Comparing metallicities and compositions at different galactic latitudes, it has been found that while the $[\alpha / \mathrm{Fe}]$ remains roughly constant and metal-poor stars show a remarkable homogeneity in the bulge (Lecureur et al. 2007; Johnson et al. 2011; Gonzalez et al. 2011), the most metal-rich stars near the galactic plane disappear at higher latitudes (see Zoccali et al. 2008; Lecureur et al. 2007; Babusiaux et al. 2010, among others). Further, the younger population is associated with the bar (Babusiaux et al. 2010, and references therein). These differences could be an indication that younger, more metal-rich stars in the bulge define a smaller region than metalpoor ones (see also Robin et al. 2012). Otherwise, Johnson et al. (2012) explored the link between bulge and thick disk formation and found diverging behaviors in the cases of $[\mathrm{Na} / \mathrm{Fe}]$ and $[\mathrm{La} / \mathrm{Fe}]$, which results in the bulge resembling more the spheroid.

In the bulges of external galaxies, similar conclusions have been reached. Stellar population studies suggest that in many cases a secondary stellar population is superimposed on an 
older one (Ellis et al. 2001; Thomas \& Davies 2006; Carollo et al. 2007), and that the two populations are kinematically distinguishable, with the old population having spheroid-like kinematics, while this secondary population is more disk-like; see, for example, Prugniel et al. (2001), and the results from the SAURON project (Peletier et al. 2007; Erwin 2008). Bulge stellar masses are generally dominated by the old populations, with the young ones contributing less than a $25 \%$ in most cases (MacArthur et al. 2009), although Kormendy et al. (2010) point out that significant numbers of local massive spiral galaxies appear to have dominant pseudo-bulges.

Jablonka et al. (2007) conclude that most external bulges in their observed sample are more metal rich and have lower $[\alpha / \mathrm{Fe}]$ enhancement in their central regions than in their outer parts, a result consistent with Moorthy \& Holtzman (2006), and with Milky Way bulge results. MacArthur et al. (2009) find a wide range of gradients, both positive and negative, allowing for different bulge formation mechanisms (see also Morelli et al. 2012). A complementary piece of information are age gradients, where most authors find that the central regions of external bulges are younger than the outer ones (e.g., Moorthy \& Holtzman 2006; Jablonka et al. 2007; MacArthur et al. 2009; Sánchez-Blázquez et al. 2011). Note, however, that the last authors have also found negative age gradients in some cases.

Summarizing, it would appear that bulge formation and evolution in a cosmological context has to account for a duality in stellar populations, and for the coexistence of spheroid-like features with disk-like ones, while some particularities may be related to minor merger events (see discussion in Combes 2009). Theoretical models focusing on the physical processes responsible for bulge properties have a long history, a sample of which is presented below. Generally, the classical type is thought to originate from fast gas collapse at high redshift, or from gas clumps in a proto-disk that are driven to the central regions by dynamical friction, while instabilities of the disk, perhaps associated with bars, are believed to result in the formation of pseudo- and/or boxy bulges.

Metal enrichment in bulges was first analytically studied through pure chemical evolution models. Matteucci \& Brocato (1990) first predicted an $[\alpha / \mathrm{Fe}]$ enhancement at bulges as a consequence of the different SNe I and II nucleosynthetic time-scales, observationally confirmed by McWilliam \& Rich (1994). Mollá et al. (2000) used a multiphase multi-zone chemical evolution model and provided solutions to bulge chemical abundances and spectral indices. Ferreras et al. (2003) found that very short infall timescales are required for bulges. These models provided the basis of later developments to be implemented in hydrodynamical simulations, and, at the same time, gave us clear insights into some of the physical processes involved.

On the other hand, purely collisionless numerical studies of bulge formation have focused on morphology and dynamics by analyzing disk evolution in pre-prepared simulations. Such studies showed that spheroidal-like bulges cannot be formed through bar-buckling instabilities (Debattista et al. 2004), while disk-like and boxy/peanut bulges are different in their nature, although both classes are associated with the presence of a bar (Athanassoula 2005). On the other hand, Eliche-Moral et al. (2011) analyze the effect of minor mergers on the inner part of disk galaxies, finding this process to be efficient in forming rotationally supported stellar inner components, i.e., disks, rings, or spiral patterns. Hopkins et al. (2010) use a different approach, by constructing semi-empirical models based on observationally motivated halo occupation numbers from the Millenium simulation, aiming to quantify the relative effect of galaxy mergers on bulge formation. They find major mergers to be the dominant mechanism for $L_{*}$ bulge and spheroid formation and assembly, while minor mergers contribute relatively more in lower mass systems.

The first numerical studies of bulge formation using dissipative collapse by Samland \& Gerhard (2003) model the formation of a large disk inside a spinning $(\lambda=0.05)$, growing dark matter (DM) halo, with an added accretion history taken from the large-scale simulations of the GIF-VIRGO consortium (Kauffmann et al. 1999). Chemical evolution is followed through two fiducial elements tracing the fraction of heavy elements produced by type Ia or type II SNe. The resulting bulge consists of at least two stellar sub-populations, an early collapse population and another one that formed later in the bar. Nakasato $\&$ Nomoto (2003) presented results of the evolution of a spherical $3 \sigma$ top-hat overdense region of $1.4 \mathrm{Mpc}$ co-moving radius in rigid rotation evolved with a gravohydrodynamical code. Again the nucleosynthetic yields of type Ia or type II SNe are used, tracing the chemical enrichment in metallicity, $\mathrm{O}$ and Fe. Their results suggest that bulges consist of two chemically different components; one that has formed quickly through a sub-galactic merger in the proto-galaxy, and another one that formed gradually in the inner disk. Kobayashi \& Nakasato (2011) further developed this latter chemical evolution implementation, and used it to simulate a Milky-Way-like galaxy. Their kinematic and chemical results follow closely the observed properties of the Galaxy halo, bulge, and thick disk. These simulations, however, do not take into account the cosmological gas infall.

Few studies on bulge formation have been made within a fully cosmological context. Tissera \& Dominguez-Tenreiro (1998) and Governato et al. (2009) have studied the effects of mergers on classical bulge stellar populations. Guedes et al. (2011) have run the highest resolution simulation up-to-now of Milky-Way-like disk formation, and found realistic disk and bulge properties, but their focus on the details of bulge formation is relatively minor. Okamoto (2012) finds that pseudo bulges form, in his simulated Milky Way galaxies, by rapid gas supply at high-redshift, with their progenitors observable as high-redshift disks, and that this occurs prior to formation of the final disk. Brook et al. (2012b) obtained a lower mass late-type disk galaxy which has a bulge that grows from $z=1$ mainly through purely secular processes. Other authors have put more emphasis on metal enrichment. For example, Rahimi et al. (2010) run a fully cosmological simulation with the GCD+ code (Kawata \& Gibson 2003), which incorporates chemical enrichment both by SNe Ia (Iwamoto et al. 1999; Kobayashi et al. 2000) and SNe II (Woosley \& Weaver 1995), as well as mass loss from intermediate-mass stars. The code does not include a mechanism to diffuse metals between gas particles, resulting in an artificially high spread in the metallicity distributions, but robust averages. Their results underline the importance of mergers in bulge formation and their possible kinematic implications, the dependence of metal content on age, and the existence of accreted stars within the bulge.

Thus, it would appear that a variety of conclusions are being drawn from different groups using different codes and different physical models for their galaxy formation simulations. By analyzing in a unified manner (measuring the relevant properties with the same pipeline) simulated disk galaxies that are run with different codes and different physical prescriptions and merger histories, in this study we hope to shed light on what 
Table 1

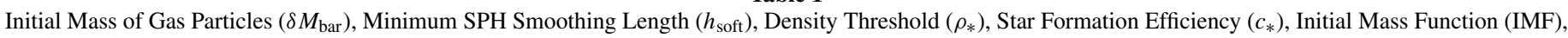
SN Feedback $\left(E_{\mathrm{SN}}\right)$, Bulge Radius $\left(r_{\text {bulge }}\right)$, Stellar $\left(M_{*}\right)$, and Gas $\left(M_{\text {gas }}\right)$ Mass of the Simulated Bulges (at $\left.z=0\right)$, and Periodic Box Length $\left(L_{\text {box }}\right)$

\begin{tabular}{|c|c|c|c|c|c|c|c|c|c|c|}
\hline Object & $\begin{array}{c}\delta M_{\mathrm{bar}} \\
\left(10^{5} M_{\odot}\right)\end{array}$ & $\begin{array}{c}h_{\mathrm{soft}} \\
\left(h^{-1} \mathrm{kpc}\right)\end{array}$ & $\begin{array}{c}\rho_{*} \\
\left(\mathrm{~cm}^{-3}\right)\end{array}$ & $\begin{array}{c}c_{*} \\
(\%)\end{array}$ & IMF & $\begin{array}{c}E_{S N} \\
\left(10^{51} \mathrm{erg}\right)\end{array}$ & $\begin{array}{l}r_{\text {bulge }} \\
(\mathrm{kpc})\end{array}$ & $\begin{array}{c}M_{*} \\
\left(10^{10} M_{\odot}\right)\end{array}$ & $\begin{array}{c}M_{\text {gas }} \\
\left(10^{8} M_{\odot}\right)\end{array}$ & $\begin{array}{c}L_{\mathrm{box}} \\
(\mathrm{Mpc})\end{array}$ \\
\hline G-1578411 & 0.2 & 0.15 & 9.4 & 1.7 & Kroupa93 & 1.0 & 1.30 & 0.83 & 1.64 & 34 \\
\hline G-1536 & 1.9 & 0.15 & 9.4 & 3.3 & Chab03 & 1.0 & 2.10 & 0.96 & 5.92 & 68 \\
\hline HD-5004A & 3.94 & 0.2 & 6 & 1.0 & Salp55 & $\ldots$ & 1.00 & 1.50 & 3.67 & 10 \\
\hline HD-5004B & 3.94 & 0.2 & 10 & 0.8 & Salp55 & $\ldots$ & 1.55 & 1.60 & 5.33 & 10 \\
\hline HD-5103B & 3.78 & 0.2 & 12 & 0.8 & Salp55 & $\ldots$ & 1.73 & 1.47 & 4.61 & 10 \\
\hline
\end{tabular}

Notes. The mass values correspond to the position selection. The P-DEVA runs use a fixed mass for the baryonic particles, equal to $\delta M_{\text {bar }}$.

processes of bulge formation, and subsequent bulge properties, are common within such simulations. Specifically, we analyze the bulges of three of the more massive galaxies presented in Doménech-Moral et al. (2012), run with the P-DEVA (Serna et al. 2003; Martínez-Serrano et al. 2008) code, and those of two GASOLINE galaxies described in Brook et al. (2012b) and Stinson et al. (2012), with the aim of deciphering the patterns of bulge formation by focusing in the properties of their stellar populations.

Doménech-Moral et al. (2012) analyze disk formation in a cosmological context by running zoom simulations with P-DEVA. This code (Martínez-Serrano et al. 2008) incorporates a statistical implementation of chemical enrichment based on Talbot \& Arnett (1973), including chemical feedback both by SNe Ia and SNe II, as well as mass loss from intermediatemass stars, involving 11 elements. Radiative cooling takes into consideration the full element distribution at each point and time through a particular implementation of the dimension reduction regression (Weisberg 2002), while an SPH metal diffusion term mimics turbulent effects (Monaghan 2005). They have produced disk systems whose different components (thin and thick disk, halo, bulge) have properties nicely consistent with observations, for example, they have $g$-band $B / T$ ratios between 0.13 and 0.36 . In particular, bulge metallicity and $[\alpha / \mathrm{Fe}]$ distributions show bimodal patterns, which they interpret as resulting from fast and slow modes of star formation. The simulations run with P-DEVA assume that supernova feedback works on subgrid scales and results in a low star formation efficiency, which is thus used as an input parameter (see discussion in Agertz et al. 2011) that implicitly mimics the energetic feedback.

On the other hand, the simulations run with the GASOLINE code have explicit feedback from massive stars which drives large-scale outflows. The two particular GASOLINE simulations were chosen for this study because (1) G-1578411 was shown to have a bulge which is mostly formed after the merger epoch. It is the late-type simulated disk galaxy from Brook et al. (2012b). A secular bulge grows between $z=1$ and $z=0$, driven at least partly by a bar. The final bulge to total light ratio is $B / T=0.21$. (2) G-1536 is the simulated $L^{*}$ galaxy run by our MaGICC program that matches the widest range of observed galaxy properties (see Stinson et al. 2012 and SG5LR in Brook et al. 2012a; see also Calura et al. 2012). It has a $B / T$ ratio of 0.35 .

We use this suite of five inhomogeneous simulated disk galaxies to analyze the mass-weighted three-dimensional shape, size, and kinematics of the bulges, as well as their mass-weighted age, metallicity, and chemical composition. More specifically, we first show that the simulated bulges consist of roughly two stellar populations (old and young) whose properties are correlated with their shape, kinematics, metallicity, and composition, in line with the observational results we have previously described. In view of this agreement with observations, we investigate the physical processes underlying bulge formation and particularly, their relation with the dynamics of the cosmic web at high redshift, and of their host galaxies at low $z$ s.

An interesting aspect of this work is to clarify to what extent the formation mechanisms proposed for massive ellipticals also apply to the formation of bulges, as suggested by similarities in observed properties (e.g., Franx 1993; Peletier et al. 1999; Carollo et al. 2001; Bureau 2002; Peletier 2008). In this regard, let us recall that the characteristics of mass assembly and star formation rate (SFR) histories in ellipticals can be interpreted in terms of dark halo dynamics and its consequences. Indeed, analytical models as well as $N$-body simulations show that two different phases can be distinguished along the halo mass assembly process (Wechsler et al. 2002; Zhao et al. 2003): (1) first a violent fast phase with high-mass aggregation rates, resulting from collapse-like and merger events, and (2) later on a slow phase with much lower mass aggregation rates. Small box hydrodynamical simulations (Domínguez-Tenreiro et al. 2006) as well as larger box ones (Oser et al. 2010; Domínguez-Tenreiro et al. 2011) confirmed this scenario as well as its implications on elliptical properties at low $z$ (Cook et al. 2009). This scenario nicely explains apparently paradoxical observational data of elliptical galaxies.

The paper is structured as follows. In Section 2, we describe the details of the simulations. The criteria used to select bulge stars are discussed in Section 3. In Section 4, we construct the star formation histories of the bulges and define the selection criteria for the old and young stellar components. In Section 5 we discuss their shapes and kinematics, and in Section 6 their chemical composition. The origin of the bulge stars in the framework of host galaxy and cosmic-web dynamics is given in Section 7. Finally, in Section 8 we draw our conclusions after a brief summary of our findings and discuss the bulge formation scenario that emerges from our simulations.

\section{THE SIMULATIONS}

The main properties and resolution of our five simulated galaxies are shown in Table 1. All galaxies have previously appeared in the literature, where more details can be garnered. Here we outline the main features of the codes and simulations.

\subsection{P-DEVA}

We use the OpenMP parallel version of the DEVA code (Serna et al. 2003), which includes the chemical feedback and cooling methods described in Martínez-Serrano et al. (2008), and in which the conservation laws (e.g., momentum, energy, angular momentum, and entropy) hold accurately (Serna et al. 2003). Full details of the recipes used are found in Doménech-Moral et al. (2012) where the simulations are first presented. The 
star formation recipe follows a Kennincutt-Schmidt-like law with a given density threshold, $\rho_{*}$, and star formation efficiency $c_{*}$. In line with Agertz et al. (2011), we implement inefficient SF parameters (see Table 1), which implicitly account for the regulation of star formation by feedback energy processes by mimicking their effects, which are assumed to work on sub-grid scales.

The chemical evolution implementation (Martínez-Serrano et al. 2008) accounts for the dependence of radiative cooling on the detailed metal composition of the gas, by means of a fast algorithm based on a metallicity parameter, $\zeta(T)$. The code also tracks the full dependence of metal production on the detailed chemical composition of stellar particles (Talbot \& Arnett 1973), through a $Q_{i j}$ formalism implementation of the stellar yields, for the first time in SPH. The delayed gas restitution from stars has been implemented through a probabilistic approach, which reduces statistical noise when compared with previous approaches, and therefore allows for a fair description of element enrichment at a lower computational cost. Moreover, the metals are diffused in such a way as to mimic the turbulent mixing in the interstellar medium.

The simulations use the cosmological "zoom-in" technique, with high-resolution gas and DM in the region of the main object. The cosmological parameters of a $\Lambda$ CDM model were assumed $\left(\Omega_{\Lambda}=0.723, \Omega_{m}=0.277, \Omega_{b}=0.04\right.$, and $\left.h=0.7\right)$, in a $10 \mathrm{Mpc}$ per side periodic box. Stellar masses are distributed according to the Salpeter initial mass function (IMF; Salpeter $1955)$, with a mass range of $\left[M_{l}, M_{u}\right]=[0.1,100] M_{\odot}$.

\subsection{GASOLINE}

The GASOLINE galaxies are cosmological zoom simulations derived from the McMaster Unbiased Galaxy Simulations (MUGS; Stinson et al. 2010). In G-1578411, the initial conditions (ICs) are scaled down, so that rather than residing in a $68 h^{-1}$ Mpc cube, it is inside a cube with $34 h^{-1} \mathrm{Mpc}$ sides, while G-1536 uses the same ICs as in the MUGS runs.

When gas becomes cool $(T<15,000 \mathrm{~K})$ and dense $\left(n_{\text {th }}>9.3 \mathrm{~cm}^{-3}\right)$, it is converted to stars according to a Kennincutt-Schmidt-like law with the SFR $\propto \rho^{1.5}$. Effective SFRs are determined by the combination and interplay of $c_{\star}$ and feedback, $c_{\star}$ is ultimately the free parameter that sets the balance of the baryon cycle off cooling gas, star formation, and gas heating. Stars feed energy back into surrounding gas. Supernova feedback is implemented using the blastwave formalism (Stinson et al. 2006) and deposits $10^{51}$ erg of energy into the surrounding medium at the end of the stellar lifetime of every star more massive than $8 M_{\odot}$. Energy feedback from massive stars prior to their explosion as supernovae has also been included. Without the ability to resolve the details, we employ a relatively crude thermal implementation of radiation feedback from massive stars, with the aim being able to mimic their most important effects on scales that we resolve, i.e., to regulate star formation, enhance inhomogeneity, and to allow the expansion of the SNe-driven super-bubbles which drive outflows. To mimic the weak coupling of this energy to the surrounding gas (Freyer et al. 2006), we inject pure thermal energy feedback, which is highly inefficient in these types of simulations (Katz 1992; Kay et al. 2002). We inject $10 \%$ of the available energy during this early stage of massive star evolution, but $90 \%$ is rapidly radiated away, making an effective coupling of the order of $1 \%$.

The two simulations have different IMFs, with G-1536 having a factor of $\sim 2$ more massive stars (Chabrier 2003) than
G-1578411 (Kroupa et al. 1993), meaning that G-1536 is less efficient at turning baryons into stars.

Ejected mass and metals are distributed to the nearest neighbor gas particles using the smoothing kernel (Stinson et al. 2006). Literature yields for SNII (Woosley \& Weaver 1995) and SNIa (Nomoto et al. 1997) are used. Metals are diffused by treating unresolved turbulent mixing as a shear-dependent diffusion term (Shen et al. 2010), allowing proximate gas particles to mix their metals. Metal cooling is calculated based on the diffused metals.

\section{BULGE SELECTION CRITERIA}

The classical method of separating the contribution of the inner region from the disk rests on fitting the light radial profile of the spiral galaxy with two (or more) components (e.g., exponential disk + a Sérsic profile). However, this method does not provide any way in which individual stellar particles can be assigned to the inferred galaxy components. Therefore, we make a kinematic separation of bulge and disk stars using clustering algorithms. For each star particle, we computed $J_{p}$, the angular momentum in the plane of the disk, $J_{z}$, the angular momentum perpendicular to the disk, and $J_{c}$, the angular momentum of a particle with the same binding energy $(E)$, moving in a circular orbit at the same radius. Each stellar particle within the virial radius is then dynamically defined by three variables: $J_{z} / J_{c}$, $J_{p} / J_{c}$, and binding energy $(E)$. These variables are normalized to $[0,1]$ in order to give equal weight to all variables, and then fed to the clustering algorithm, which requires as prior only the number of clusters (we chose $n=2$ for bulge + disk). The normalization assumes a linear mapping between $\left[X_{\min }, X_{\max }\right]$ and $[0,1]$ (with $J_{z} / J_{c}, J_{p} / J_{c}$ and $E$ as $X$ ), which is appropriate if the histogram of $X$ does not have extremely extended tails. We considered the distance in the phase space to be the Euclidean one. The algorithm, starting from an initial random partitioning into $n$ clusters, iteratively searches for the partitioning which would minimize the intracluster distance. In Figure 1, we show the output of the clustering algorithm in the form of the histograms of $J_{z} / J_{c}, J_{p} / J_{c}$, and $E$ for disk, spheroid and galaxy stars in the case of G-1536. As expected, the spheroid stars peak at $J_{z} / J_{c}=0$ in accordance with a system sustained by velocity dispersion, while the disk ones peak closer to $J_{z} / J_{c}=1$. In the $J_{p} / J_{c}$ histrograms, the disk shows a peak at $\sim 0.1$, characteristic of a system whose particles move a little perpendicular to the disk plane, although some of them can have an important vertical motion which shows up as the tail extending to $J_{p} / J_{c} \sim 0.6$.

Once the dynamical decomposition has been done, the projected stellar mass density profiles of the disk (thin plus thick) and spheroid naturally provide us with the radial cut necessary to delimit the bulge, in the form of the radius, $r_{\text {bulge }}$, where the two intersect. Within this cut the mass contribution of the disk stars is much smaller than the spheroid, therefore minimizing the contamination from disk stars when determining the bulge global properties. An example of projected stellar mass density for the disk, spheroid, and galaxy stellar particles is given in Figure 2 for G-1536, as well as the position of the radial cut we use to define the bulge region.

Although a dynamical separation of galaxy components is of great help in the analysis of galaxy structure formation, from an observational point of view the different dynamical components of the central galactic regions are usually hard to disentangle. For this reason, we also use an observationally based selection for the bulge stars, in the form of a simple radial cut at $r_{\text {bulge }}$. 

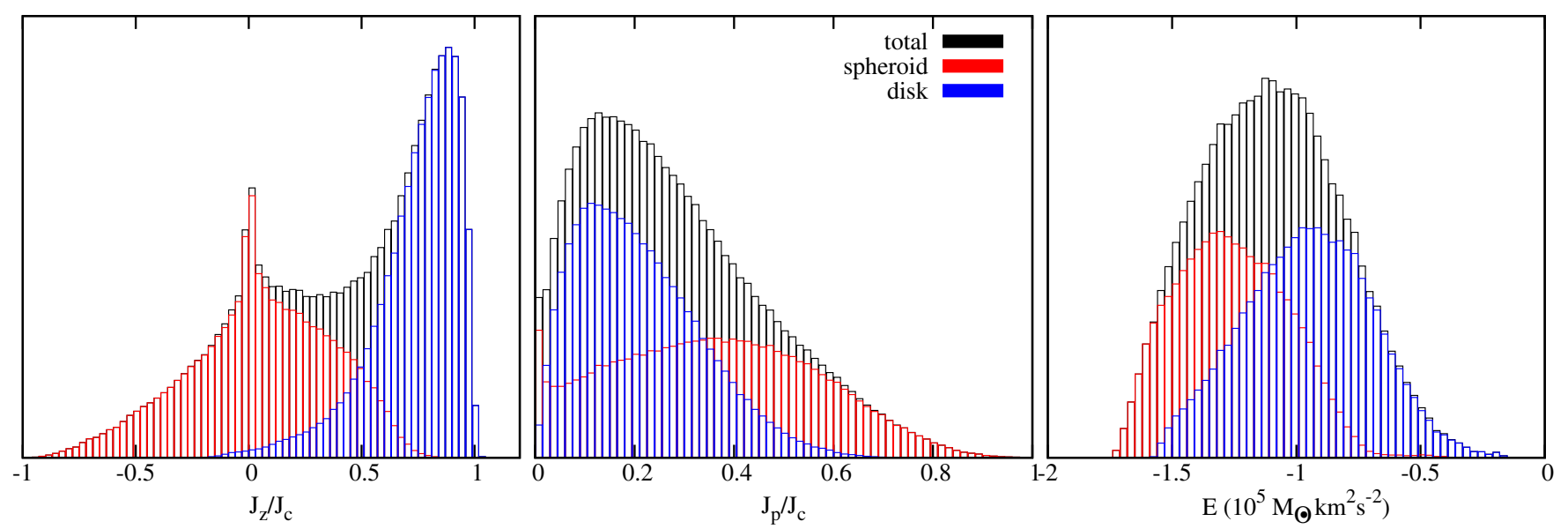

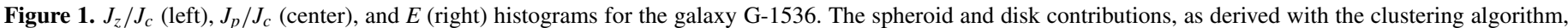
are given in red and blue, respectively. The histograms considering all the galaxy stellar particles are given in black.

(A color version of this figure is available in the online journal.)

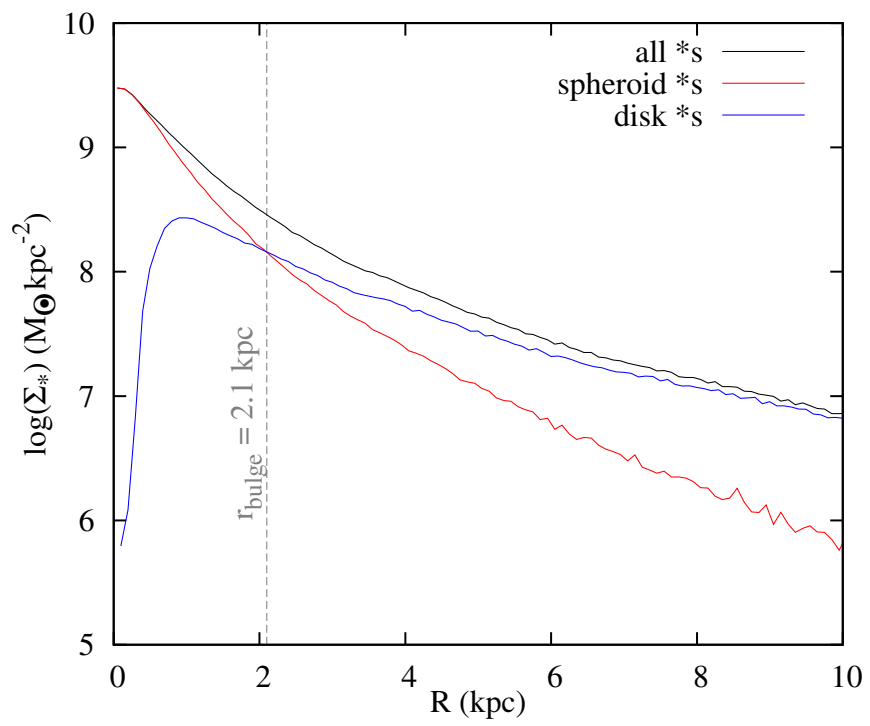

Figure 2. Projected stellar mass density for the kinematic disk (blue curve) and spheroid (red curve), and all stellar particles (black curve) of galaxy G-1536. The gray dashed line gives the position of the radial cut used to define the bulge region.

(A color version of this figure is available in the online journal.)

Summing up, we use two bulge selection criteria: (1) the stars within a sphere of radius $r_{\text {bulge }}$, hereafter position selection, and (2) the stars within a sphere of radius $r_{\text {bulge }}$ that at the same time belong to the galaxy spheroid according to the kinematic decomposition, hereafter kinematic selection. In Table 1, we give $r_{\text {bulge }}$, the total stellar and gas mass of each bulge at $z=0$, as well as the mass resolution for each simulation.

\section{BULGE POPULATIONS}

To analyze the bulge stellar populations and their link with dynamical processes, we constructed Figure 3, in which both the SFR histories of the bulge and the galaxy mass aggregation tracks (MATs) have been drawn. The MATs each give the evolution of mass inside a fix radius (right axis in each panel). Virial radii have been calculated based upon the Bryan \& Norman (1998) fitting function to determine the overdensity threshold. For the baryonic component, radii are binned in equally spaced steps in a logarithmic scale. On the right (left) panels the colored curves show the stellar (stellar plus cold gas) masses within the respective radii as a function of the universe age $\left(t_{U}\right.$ is the universe age at $\left.z=0\right)$ and redshift. MATs corresponding to radii within $r_{\text {bulge }}$ are drawn with different colors, while those at larger radii are given in cyan.

Major mergers $\left(M_{\text {secondary }} / M_{\text {main }}>0.25\right)$, minor mergers and slow accretion processes in the DM or baryonic component can be clearly identified as big or small mass jumps in the MATs, or as continuous mass increments, respectively (note the different mass scales on the right axes for the P-DEVA and GASOLINE galaxies). Two different phases are reflected in the noticeable knee-like shape of the MATs in all objects shown in Figure 3: an initial phase where the mass assembly rate is high, and then a much slower phase, when mass is more slowly acquired.

Superimposed on the same figure are the bulge SFR histories (left axis in each panel), for both position and kinematic selected bulge populations. All objects present starbursts at large redshifts, peaking between $z=3$ and $z=1$, where significant substructure is also apparent in many cases. These early star formation peaks are expected when the primordial gas suffers a violent collapse and are correlated with the knee-like feature visible in their corresponding MATs. The high-redshift SF peak is a direct consequence of the first phase of mass assembly. The simulations also show later peaks in star formation that are related to merger/accretion events.

At lower redshifts a variety of processes appear imprinted in the SFR histories: during quiescent periods, the SFR decays to an approximately constant tail at the same time as the MATs are flattened. The low tails present in all SFR histories correspond to periods of mass assembly where only minor mergers or gas accretion at slow rates show up in the MATs. All simulations also show secondary jumps in SFRs, associated with later merger events. These vary in time and in size relative to the initial starbursts, for example, HD-5103B has a relatively late $\left(t / t_{U}\right)$ accretion event that shows up in the total mass MAT (black line) as well as the build up of bulge mass and the SFR.

Some qualitative differences are apparent in the two GASOLINE runs compared to the P-DEVA ones. The initial starbursts associated with the fast phase of mass accretion are less peaked in the former, due to the affects of feedback. In G-1578411, which has the lower mass but also lower feedback of the two GASOLINE simulations, the initial starburst has a 


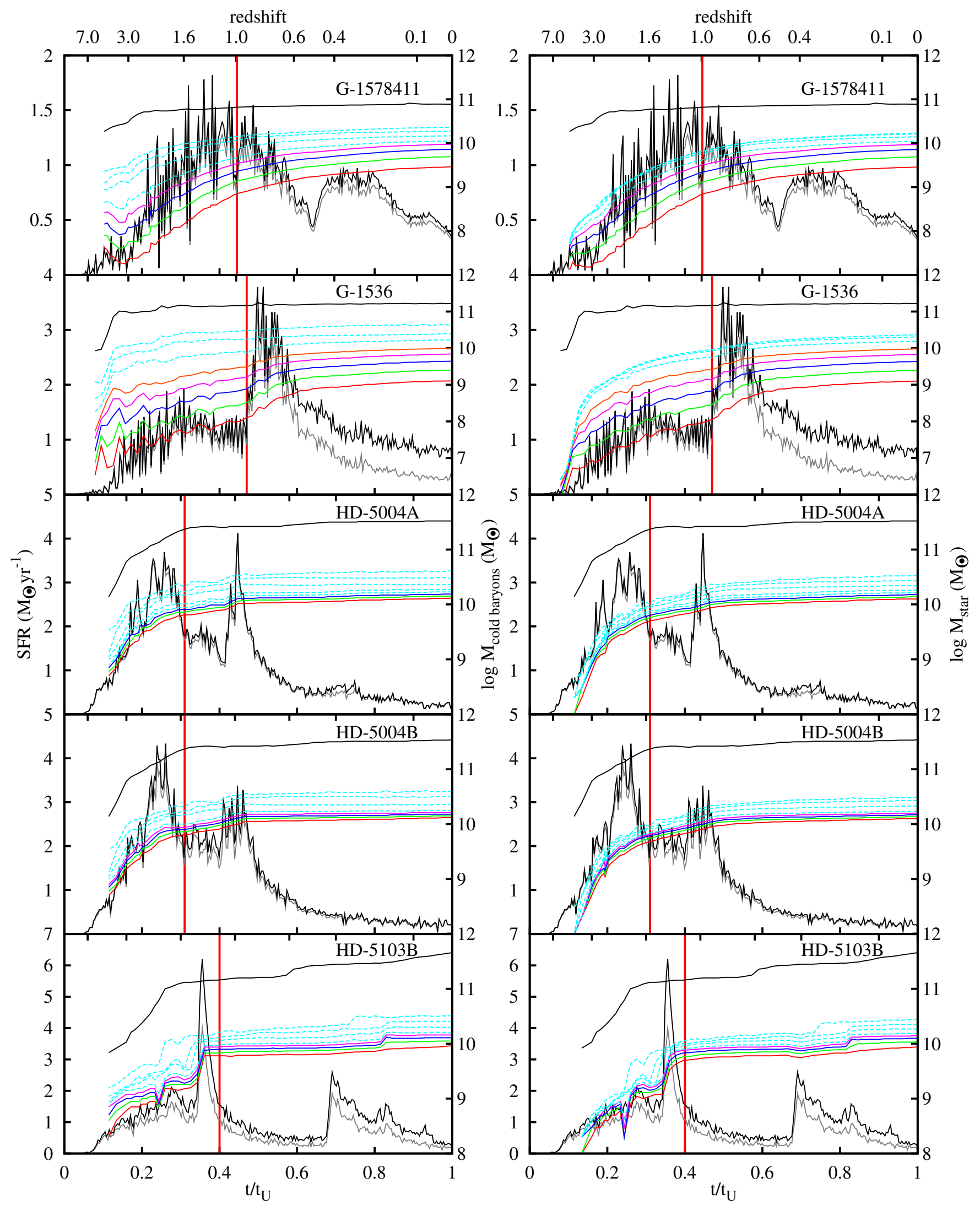

Figure 3. Bulge star formation rate comparisons for kinematic (solid gray) and position (solid black) selected bulge stars with sizes given by $r_{\text {bulge }}$. The position of the thick red vertical (values in Table 2) indicate the separation between the old and young stellar populations. The colored lines represent the mass aggregation tracks (MATs) of cold baryons (left) and stars (right) along the main branch of the merger tree for each object, the masses being computed inside fixed radii of 0.50 , 0.72 , $1.03,1.47,2.10,4.30,8.80$, and $25.80 \mathrm{kpc}$ from bottom to top. The upper solid black line is the total mass inside the virial radius (virial mass), the solid curves in red, green, blue, magenta, and orange correspond to radii roughly within the bulge, while the dashed cyan ones correspond to radii exceeding $r_{\text {bulge }}$ (see Table 1). 
Table 2

Temporal Separation Between the Distinct Star Formation Episodes for the Simulated Bulges, the Corresponding Mass Percentage of Old and Young Stars in the Position Selection, and the Sérsic Indices Derived from Fitting the Projected Stellar Mass Density of Old and Young (According to the Bulge Temporal Cut), and of All Galaxy Stellar Particles

\begin{tabular}{lcccccc}
\hline \hline Object & $t_{\text {cut }} / t_{U}$ & $z_{\text {cut }}$ & $M_{\text {old } *} / M_{*}$ & $n_{\text {old }}$ & $n_{\text {young }}$ & $n_{\text {total }}$ \\
\hline G-1578411 & 0.45 & 0.98 & 0.39 & 1.17 & 1.07 & 1.22 \\
G-1536 & 0.47 & 0.90 & 0.31 & 1.19 & 1.59 & 1.39 \\
HD-5004A & 0.31 & 1.54 & 0.43 & 2.95 & 2.09 & 2.57 \\
HD-5004B & 0.31 & 1.54 & 0.42 & 3.96 & 3.38 & 3.23 \\
HD-5103B & 0.40 & 1.12 & 0.50 & 3.49 & 3.27 & 3.68 \\
\hline
\end{tabular}

longer duration than the other simulations, while in G-1536 the initial starburst in the bulge region is more suppressed relative to the overall star formation within the bulge, due to the more efficient feedback in this simulation. Also, due to energetic feedback, the MATs of G-1578411 and G-1536 show delayed baryon mass assembly relative to their halos collapse at high zs. As a result, these objects have low central baryonic mass density, the effect being more marked at high- $z$.

Regardless of these specific differences, the correlations between MATs and SFR histories suggest that a meaningful stellar age classification can be based upon the two phases showing up in the MATs. Therefore, we classify as old bulge stellar population the stars formed as a direct consequence of the fast phase (with formation times smaller than the temporal cut drawn in thick red in Figure 3), while we globally denote the stars formed later on as young bulge stellar populations. In a quantitative sense, the temporal cuts correspond to the time from which the second derivative of the MAT $\left(r_{\text {bulge }}\right)$ becomes flat, reflecting a transition from a fast clumpy mass assembly to a slow smooth regime, without considering the MAT variations induced by low-redshift mergers, like in the case of HD-5103B. The values of the temporal cuts, $t_{\text {cut }}$, for all bulges are given in Table 2, together with the mass weight of the old population.

\section{3D SHAPES, SIZES, AND KINEMATICS}

To quantify the shape of the bulges, as well that of the old and young bulges as defined above, we show the correlations between the axis ratios of each ellipsoid of inertia in Figure 4. The inertia tensor was computed following González-García \& van Albada (2005) and subsequently diagonalized. Next, the eigenvalues $\left(\lambda_{1}>\lambda_{2}>\lambda_{3}\right)$ were used to compute the length of the principal axis $a \geqslant b \geqslant c$. Red and blue represent the old and young bulges, while black gives the average over the whole object. The filled and empty symbols correspond to the objects in the position and kinematic selection, respectively.

The objects situated on the dashed black line in Figure 4 have $b=c$, while the dash-dotted gray curves denoted by $T=0.3$ and $T=0.7$, respectively, separate the oblate objects from the triaxial and the prolate ones according to the $T$ parameter introduced by de Zeeuw \& Franx (1991) with the definition $T=\left(1-(b / a)^{2}\right) /\left(1-(c / a)^{2}\right)$. The oblate objects correspond to $c / a<0.9$ and $T<0.3$, the prolate to $c / a<0.9$ and $T>0.7$, and the rest to triaxial ones. In this perspective, we observe that both complete bulges as well as their distinct components are all oblate with the exception of the secular bulge of G-1578411 which is triaxial regardless of the selection criteria. Old and young populations are clearly segregated, either in the position or in the kinematic selections, and more separated in $c / a$ than in

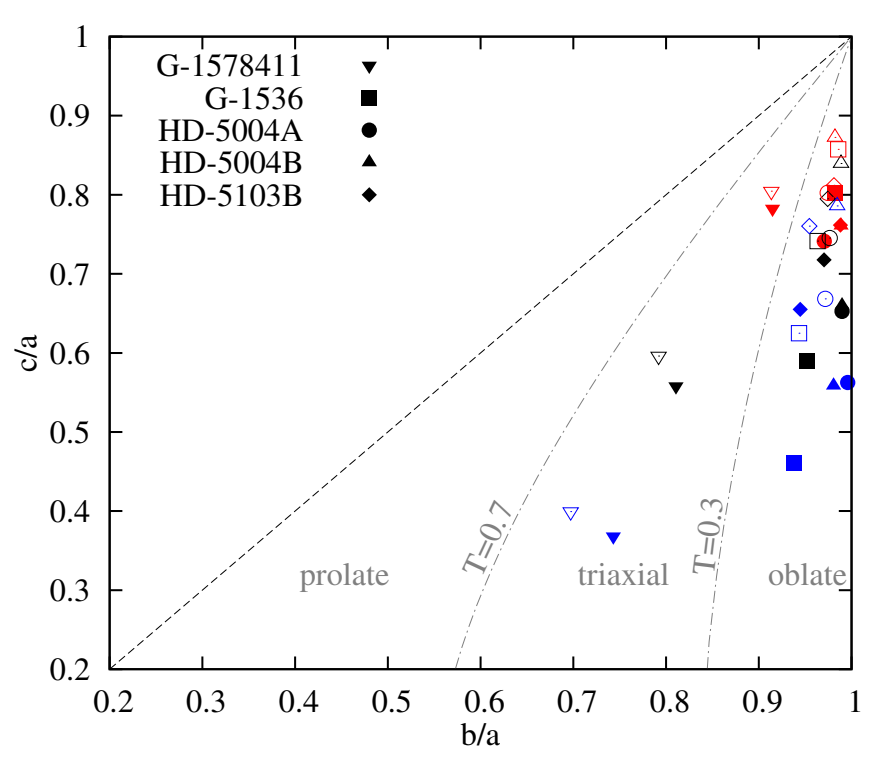

Figure 4. Axis ratios of the five spheroids. Red and blue represent the old and young bulges, while black gives the average over the whole object. The filled and empty symbols correspond to the objects in the position and kinematic selection, respectively. The dash-dotted gray curves separate prolate, triaxial, and oblate morphologies.

(A color version of this figure is available in the online journal.)

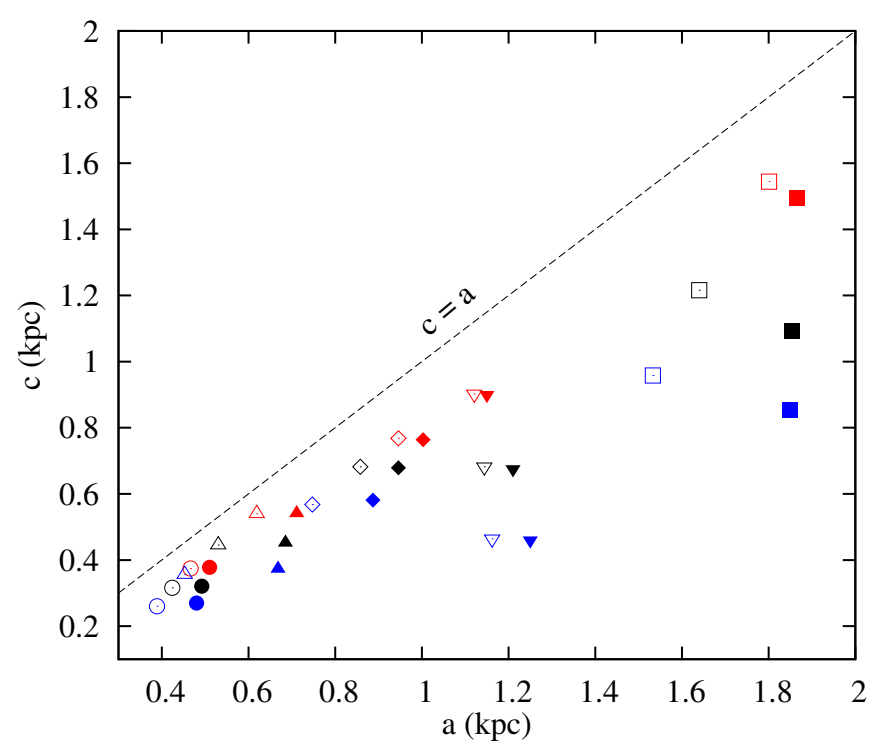

Figure 5. Minor vs. major semi axis for the five spheroids. Red and blue represent the old and young bulges, while black gives the average over the whole object. The filled and empty symbols correspond to the objects in the position and kinematic selection, respectively. The dashed line is where $c=a$. Young bulge populations are smaller than old ones, with the exception of G-1578411.

(A color version of this figure is available in the online journal.)

$b / a$. Old populations are more spheroid-like, while the young are more oblate, consistent with observations.

Figure 5 gives an idea of the sizes of the five bulges by plotting $c$ versus $a$. In all but one case (the $a$ value of G-1578411), the old bulges have larger sizes (as given by $a$ and even more by $c$ ) than the young ones, sustaining the idea that younger stellar populations occupy a smaller volume than their corresponding old counterparts. In this respect, the following inequality holds, irrespective of the selection criteria used: $\sqrt[3]{a_{y} b_{y} c_{y}}<\sqrt[3]{a_{t} b_{t} c_{t}}<\sqrt[3]{a_{o} b_{o} c_{o}}$, where $y, t$, and $o$ stand for young, total, and old, respectively. This graph shows a clear 
sequence, the size increasing from the younger to the older, with the total population in between. Also, as it was proven by the previous graph, the older bulge components are closer to be perfect spheroids (they appear closer to the dashed line for which $c=a$ ).

The difference between the results obtained using the two selection criteria are only qualitative. Indeed, the trends in shapes and sizes are the same for both selections. The kinematic-selection leads, on average, to bulge components closer to being spherically symmetric than the position selection does. This is an expected result since position selection implies the exclusion of precisely some of the particles encountered in ordered rotation which would otherwise lead to ellipsoids of inertia characteristic of more disk-like objects.

Also, the shape and size trends with stellar age are the same for the P-DEVA and GASOLINE bulges. The only difference is that GASOLINE bulges have lower mass concentrations and therefore larger values for $a$ and $c$, due to energetic feedback.

Let us now turn to the bulges kinematical analysis, including some of their classical or pseudo-bulge properties, therefore using line-of-sight (los) velocities. In order to analyze the rotational support, we align the galaxies with the $z$-axis perpendicular to the disk. Since the rotational velocity can be best observationally measured in edge-on projection, we consider two lines of sight (along the $x$ - and $y$-axes), and compute the radial profiles of the velocities $-v_{\text {los }=x}(R=y, z)$ and $v_{\text {los }=y}(R=x, z)$, respectively-along the two. The dependence of the profiles on the altitude $z$ above the galactic plane is important both when studying whether the bulge or its components are in cylindrical rotation, as well as from the point of view of comparison with observations. In the latter case, data are normally taken, due to the extinction effect in the disk plane, with long-slit or IFUs at altitudes higher than the vertical scale height of the thin disk. Considering all these observational limitations, we constructed the velocity profiles using a $z$-binning of $0.2 \mathrm{kpc}$, averaging the curves in the two hemispheres in order to minimize the statistical noise in the slits at higher latitudes where the number of particles is smaller. Complementary to the profiles of rotational velocity, we also constructed the los velocity dispersion radial profiles, $\sigma_{\operatorname{los}}(R, z)$, which are approximately flat. Given the flatness of these profiles, we considered the $\sigma_{\text {los }}(z)$ as the average of $\sigma_{\text {los }=x \mid y}(R=y \mid x, z)$ over all lines of sight $R=y \mid x,{ }^{5}$ and weighted with the number of particles in each bin $n_{R}$. Once the rotational velocity profiles were constructed, we took as $v_{\max }(z)$ the weighted average of the profile extremes. Finally, we averaged $v_{\max }(z)$ and $\sigma_{\text {los }}(z)$ over the two lines of sight, los $=x$ and $\operatorname{los}=y$.

In order to choose a representative $z$-bin for all bulges, we considered both the disk vertical scale heights as well as the bulge radial extensions. Therefore, we selected the bin with $0.4<|z|<0.6 \mathrm{kpc}$. Given this choice, within the current section, $v_{\max }$ and $\sigma_{\text {los }}$ will refer to this specific slit.

Figure 6 plots rotational support, defined as the maximal rotational velocity $\left(v_{\max }\right)$ divided by the bulge los velocity dispersion $\left(\sigma_{\text {los }}\right)$, as a function of the intrinsic ellipticity $\varepsilon=$ $1-c / a$. The dashed black curve in the graph represents oblatespheroid systems with isotropic velocity dispersion flattened only by rotation (Binney 1978) and is approximately described by $\sqrt{\varepsilon /(1-\varepsilon)}$ (Kormendy 1982).

The results show a clear trend of higher eccentricities and rotational support for the younger populations (blue symbols)

\footnotetext{
$5 y$ stands for $y$ or $x$.
}

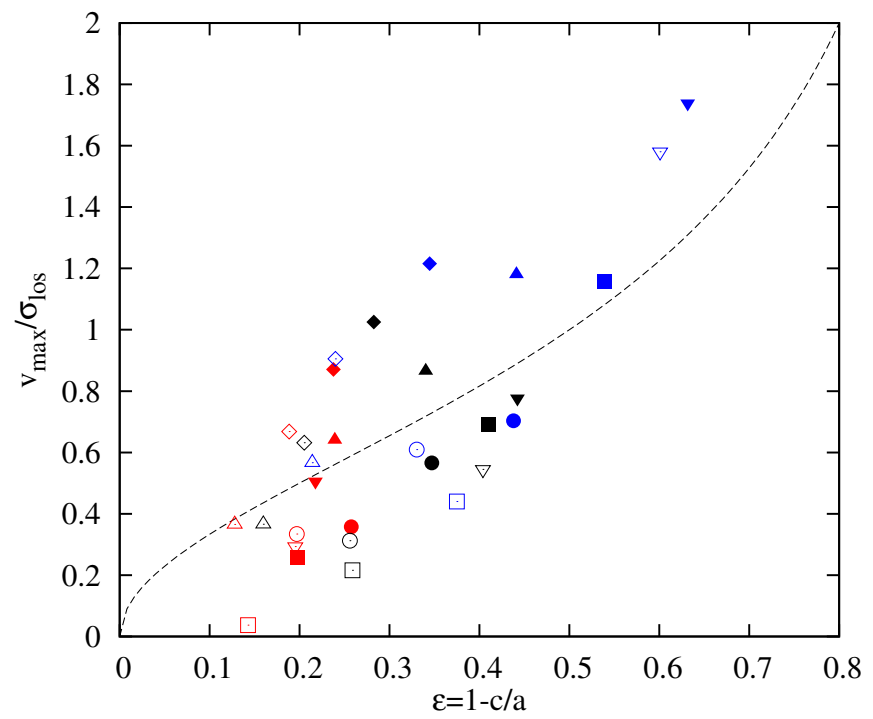

Figure 6. Rotational support vs. ellipticity. Red and blue represent the old and young bulges, while black gives the average over the whole object. The filled and empty symbols correspond to the objects in the position and kinematic selection, respectively. Young bulges are more rotationally supported and less spherical than the old populations, even when kinematically selected to exclude the disk.

(A color version of this figure is available in the online journal.)

as compared to the older ones (red symbols), while the whole bulges, in black, occupy intermediate positions. It is important to note that these patterns hold irrespective of the code used to run the simulations or the bulge selection criteria, as well as of the slit positioning. Note, however, that increasing (decreasing) the $z$ slit positioning lowers (increases) the specific values of $v_{\max } / \sigma_{\text {los }}$ for all the five bulges as well as in their distinct stellar components. Therefore, our bulges appear more classical as we move away from the galactic plane, but they always show the same trends with stellar age, with the younger component being more rotationally supported. We note that in all cases, a weighting by light rather than mass will provide increased prominence to the younger pseudo-bulge-like populations. In two cases (HD-5004A and HD-5103B in the dynamical selection), the bulge as a whole has a slightly lower value of $v_{\max } / \sigma_{\text {los }}$ even than the old population. In these cases, the bulges have velocity dispersions approximately equal to the old components, but have slightly smaller $v_{\max }$. This difference in $v_{\max }$ can be explained through misalignments and/or kinematical peculiarities.

Another parameter describing the bulge shape is the Sérsic index $(n)$. Thus, we fitted the projected stellar mass density profiles of our five galaxies with a Sérsic + an exponential disk. The data and the fits are depicted in Figure 7, while the Sérsic indices are given in Table 2. With the aim of checking whether $n$ varies if considering only the old or only the young stars, we also fitted the two stellar subpopulation of the galaxies, using for the temporal cut the limit defined with respect to the bulge region. It is important to stress that $n$ values are, to a large extent, dependent on the amount of feedback. In the case of GASOLINE galaxies, the increased feedback leads to a flattening of the projected stellar mass curves at small radii. For this reason, the Sérsic indices of G-1578411 and G-1536 are $<2$ irrespective of considering all galaxy stars or only the corresponding components, while those of HD-5004A, HD5004B, and HD-5103B (simulated without explicit energetic feedback) are $>2$. In any case, the difference between $n_{\text {old }}$ and $n_{\text {young }}$ is small in any of the simulated galaxies, with a slight 


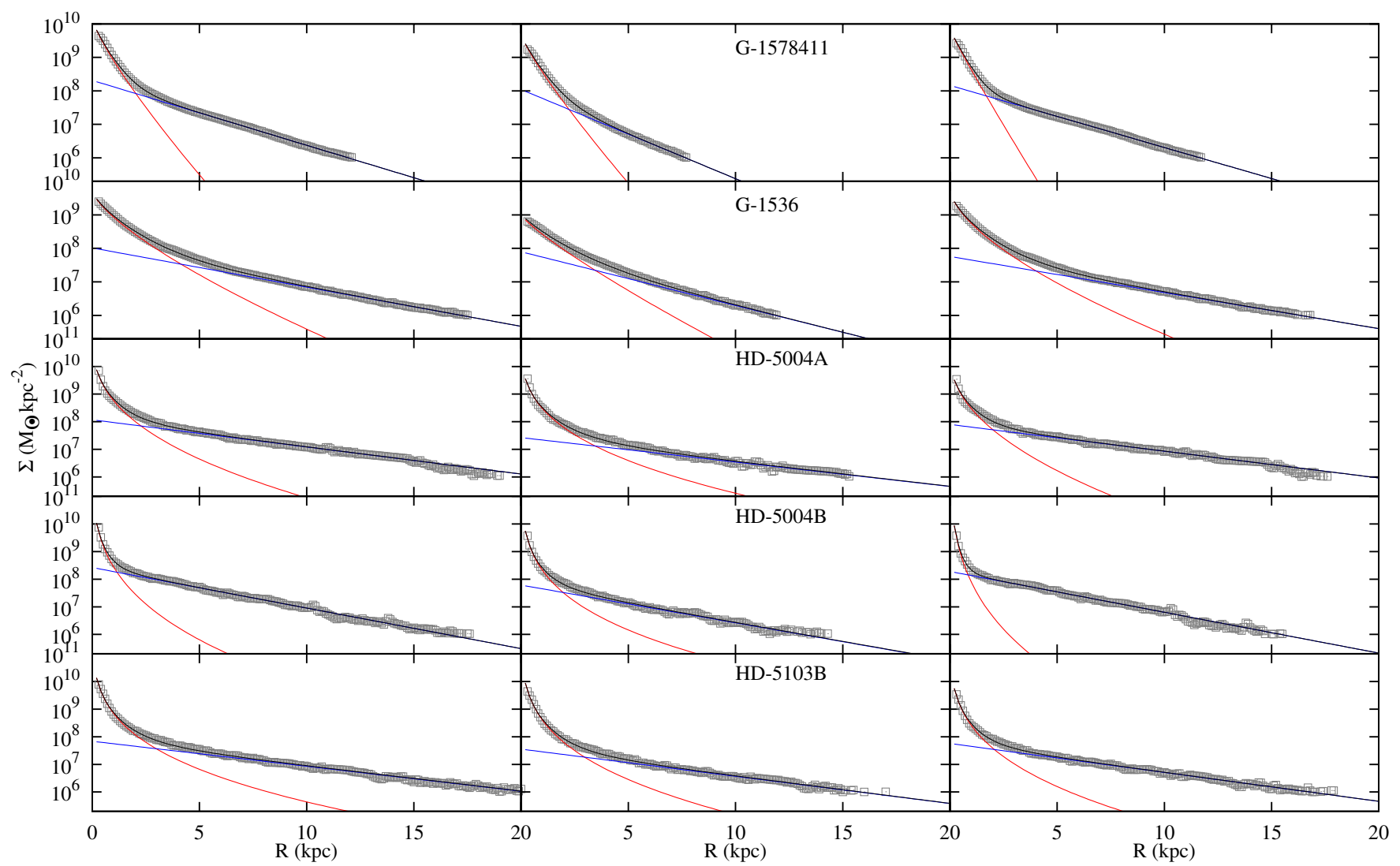

Figure 7. Sérsic + exponential disk fits of the projected mass density of galaxy stars (left panels), and of the corresponding old (central panels) and young (right panels) stellar sub-populations, separated according to the bulge temporal cut in Table 2 . The color code is gray open squares for the data, red and blue lines for the Sérsic and exponential disk contributions, and black lines for the total fit.

(A color version of this figure is available in the online journal.)

tendency for the old stars to have larger $n$ than the younger ones, with the exception of G-1536, which was simulated with a considerable amount of feedback. From an observational point of view, no definite trend with the band has been detected either; see, for example, Fisher \& Drory (2008), or more recently McDonald et al. (2011). The Sérsic index has been used, together with other parameters like rotational support, to classify bulges (Fisher \& Drory 2010). For example, Kormendy \& Kennicutt (2004) consider pseudo bulges to have $<2$, while classical ones have $>2$ (but see Graham 2011). In this respect, the three P-DEVA galaxies have classical bulges, the old bulges being more classical than the young ones ( $\left.n_{\text {old }}>n_{\text {young }}\right)$. On the other hand, the two GASOLINE ones have pseudo bulges according to this classification and show no definite trend with age. In the same perspective, the pseudo bulges of Okamoto (2012), formed at high redshift, roughly during what we define as the fast phase of mass assembly, have $<2$. However, his simulations also use energetic feedback which is at least partially responsible for the low values of $n$. Therefore, we think that further studies are needed in order to disentangle to what extent the $n$ values depend on the particular modes of mass assembly and to what extent on the amount and implementation of the feedback effects. In this respect, our study suggests that, at least for simulations, $v / \sigma$ separates more reliably the young from the old bulge than $n$, which tends to be similar for old and young stellar populations.

\section{AGES AND ABUNDANCES}

This section concerns the ages and metallicities of the old and young bulge stars at $z=0$.
Although details differ slightly, in both the P-DEVA and GASOLINE simulations trace the production and enrichment of chemical elements in broadly the same way. Newly produced elements, as by-products of stellar evolution and death, are released to the surrounding interstellar medium as increments in the metal content of nearby gas particles. Metal diffusion is implemented among gas particles with a diffusion constant, allowing for elements to mix in a given environment, mimicking what happens in a turbulent interstellar medium. Both codes consider the evolution of the following elements: H, He, C, N, O, $\mathrm{Ne}, \mathrm{Mg}, \mathrm{Si}, \mathrm{S}, \mathrm{Ca}$, and $\mathrm{Fe}$. As we are looking at broad trends here, the relatively small differences in the implementation chemistry between the codes are not critical. As a measure of metallicity we used either $[\mathrm{Fe} / \mathrm{H}]$ or the average values of these elements; also, we used $[\mathrm{Mg} / \mathrm{Fe}]$ to follow $\alpha$-element properties and checked that using $[\mathrm{O} / \mathrm{Fe}]$ does not change substantially our results. The reference values for the solar metallicities were taken from Grevesse \& Sauval (1998).

We analyze the distributions of metallicity and $\alpha$-element abundances in the different bulge populations. They are plotted in Figure 8 (central and right panels), where it can be seen that the abundance distributions of young and old bulge populations are segregated (the old stars have a lower metallicity and a higher $[\mathrm{Mg} / \mathrm{Fe}])$, giving in most cases overall two-peak $[\mathrm{Fe} / \mathrm{H}]$ and $[\mathrm{Mg} / \mathrm{Fe}]$ distributions. The slightly cleaner separation of two populations in $[\mathrm{Mg} / \mathrm{Fe}]$ space in the P-DEVA simulations as compared to the GASOLINE ones might partially be an effect of the energetic feedback in the latter, which delays the SF relatively to dynamical processes (see Figure 3 and the 

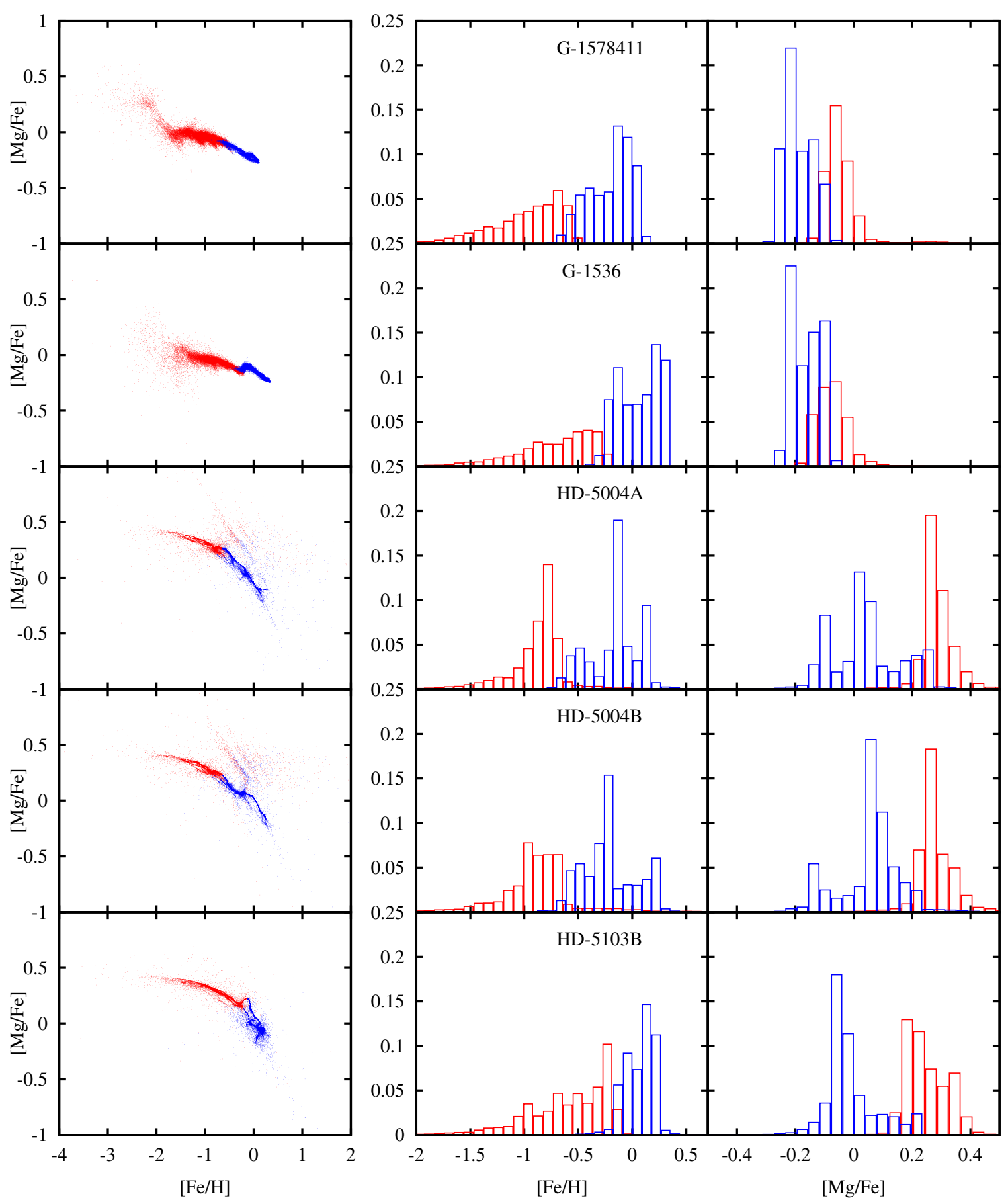

Figure 8. $[\mathrm{Mg} / \mathrm{Fe}]$ vs. $[\mathrm{Fe} / \mathrm{H}]$ (left), and the $[\mathrm{Fe} / \mathrm{H}]$ (center) and $[\mathrm{Mg} / \mathrm{Fe}]$ (right) histograms for the simulated bulges. The bin widths for $[\mathrm{Fe} / \mathrm{H}]$ and $[\mathrm{Mg} / \mathrm{Fe}]$ are 0.07 and 0.04 dex, respectively. Red and blue represent the old and young bulges.

(A color version of this figure is available in the online journal.)

corresponding comments in Section 4) and enhances the mixing, although the differences in the details of implementation of metal enrichment could also play a role, as well as in the wider ranges of $[\mathrm{Mg} / \mathrm{Fe}]$ of the P-DEVA runs. However, the important point here is that both codes result in broadly the same relative trends in metal abundances and enrichments of their old and young populations.

In this respect, we also plot $[\mathrm{Mg} / \mathrm{Fe}]$ as a function of $[\mathrm{Fe} / \mathrm{H}]$ (left panel). Again the young and old populations show up at different loci in this plot. A feature to be noted in the graphs $[\mathrm{Mg} / \mathrm{Fe}]$ versus $[\mathrm{Fe} / \mathrm{H}]$ are the slopes of the two stellar population types in the sense that the old one has a milder slope, while the young population shows a steeper one. Again, these trends are apparent in all simulations, and what is interesting is that the age selection we have used invariably separate at the "knees" of the abundance trends. This feature has to do with the different nucleosynthetic origin of $\alpha$ and Fe elements, a clean knee-like pattern being expected in stellar populations where the SFR is concentrated in an episode with a very short timescale followed by an epoch of more quiet star formation. The plateau corresponds to the early stages when $\mathrm{SNe}$ II dominate the metal enrichment, followed by a downturn to lower $[\mathrm{Mg} / \mathrm{Fe}]$ when 


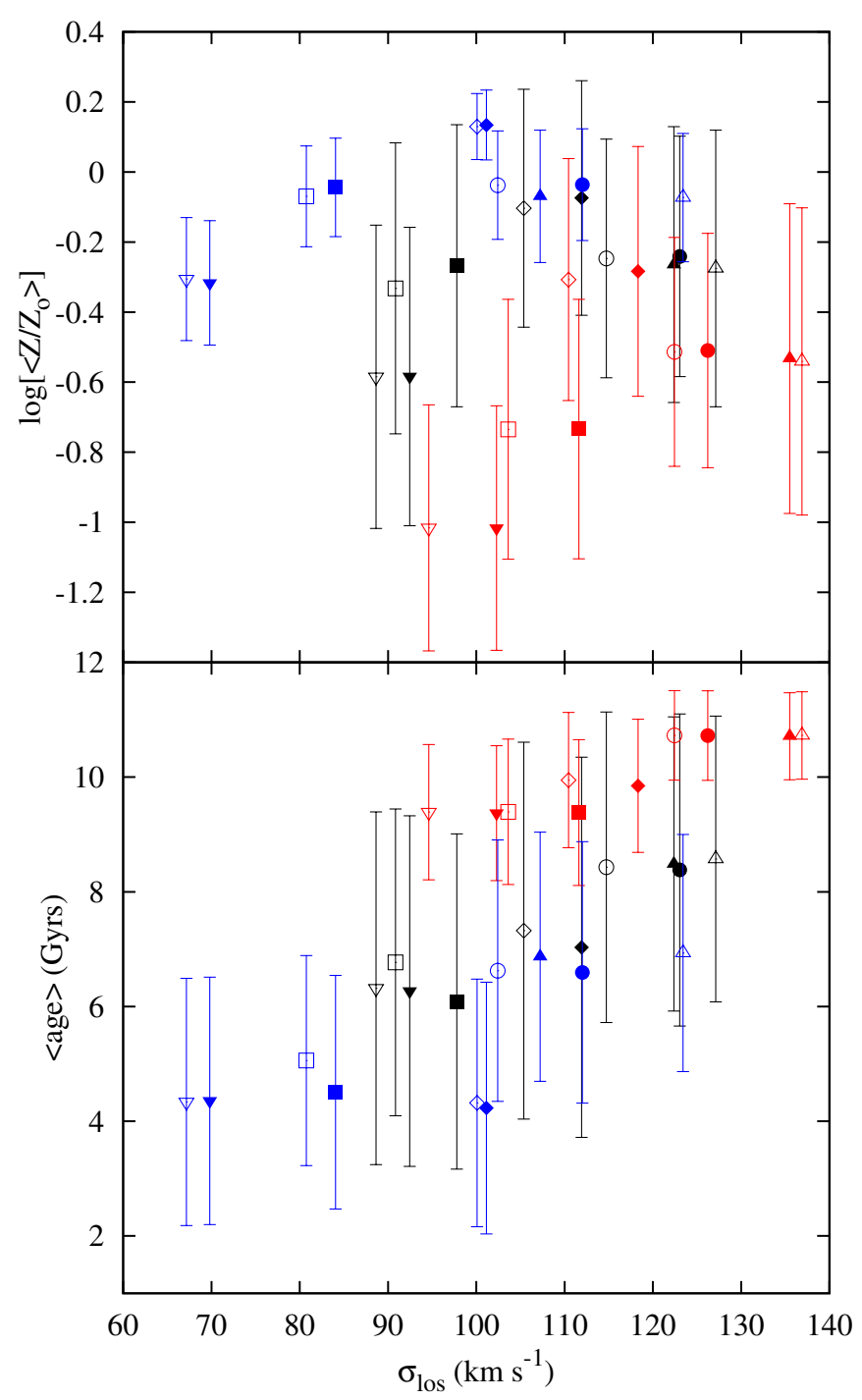

Figure 9. Average metallicity (top) and age (bottom) of the distinct bulge components vs. los velocity dispersion. Red and blue represent the old and young bulge, while black gives the average over the whole object. The filled and empty symbols correspond to the objects in the position and kinematic selection, respectively.

(A color version of this figure is available in the online journal.)

enough time has elapsed since the high-redshift starburst that SNe Ia come into play (see Wyse 1999 and references therein).

These results on the chemical composition of bulge populations agree with recent observations of the Milky Way Bulge, like those of Babusiaux et al. (2010), who found two distinct populations in Baade's Window, one old, metal-poor component with kinematics of a spheroid and a younger, metal-rich one displaying kinematic characteristics similar to a bar. Otherwise, Ness et al. (2012) extended this work by enlarging the number of windows, finding consistent results (see Section 1 for more details).

Figure 9 gives the averaged metallicities and ages of the distinct stellar populations as functions of the los velocity dispersion. In this case, $\sigma_{\text {los }}$ was computed in the same way as in Section 5, the only difference being that no $z$-binning was used. First of all, we note that a clear population segregation stands out in both panels, irrespective of the code or the selection criteria employed. In the upper panel, the metallicity appears to be increasing with the velocity dispersion, when considering all five bulges. However, if discarding the G-1578411 bulge (bottom-up triangles), $\langle Z\rangle$ seems to be almost constant with $\sigma_{\text {los }}$. On the other hand, if considering only the P-DEVA bulges, $\langle Z\rangle$ decreases with $\sigma_{\text {los }}$. These trends should be considered with caution, given the inhomogeneity of our sample. In the age- $\sigma_{\text {los }}$ plot, we get a positive slope, in accordance with the findings of MacArthur et al. (2009). Taking $\sigma_{\text {los }}$ as a measure of the objects mass (Binney \& Tremaine 1987; see Table 1 for the bulge stellar masses), it can be noted that the more massive objects also have the older average overall populations.

\section{BULGES WITHIN THE COSMIC WEB}

We trace the evolution of particles in the bulge at low $z_{\text {low }}=0$ to their origins at $z_{\text {in }}=10$, following them at 60 different redshifts in between. In Figure 10, we plot the evolution of the baryons that form the bulge stars of HD-5004A at $z=0$ (left and central graphs) at four zs (top to bottom) representing relevant consecutive events in this bulge formation, as well as the object's surroundings (right graphs) at each redshift. ${ }^{6}$ In the other simulations, this sequence of evolution is similar enough that we can use this as a representative example. The left sequence aims at illustrating the differences in the space configurations that old $z=0$ bulge-to-be stars (red points) form along their evolution as compared to those of young $z=0$ bulgeto-be stars (blue). The emphasis here is on the two population types, irrespective of whether they are gas or stars at the universe age corresponding to the plot. The central sequence takes into consideration the bulge-to-be stellar particle properties at the $z$ s plotted, namely points are green for cold gaseous particles $\left(T(z)<10^{6} \mathrm{~K}\right)$, black for hot ones $\left(T(z)>10^{6} \mathrm{~K}\right)$, and red and blue for stellar particles, depending on their time of birth according to Table 2. Its aim is to illustrate the differences in the birthplaces of the two bulge stellar populations relative either to the cosmic web (at high $z \mathrm{~s}$ ) or to the protogalaxy components (at lower zs), as well as their evolution toward their final configuration at $z=0$. Finally, in the right panels we plot all the particles in the same volumes, and with the same color code as in the central one, in order to compare the dynamical processes of the bulge-to-be stellar particles with those of the surrounding mass elements causing them.

We first analyze the sequence of bulge formation events in terms of the cosmic-web element dynamics (central panels). Before $z=3.5$ mass piles up in caustics, ${ }^{7}$ where already some nodes show up. At this redshift, star formation is already triggered in the densest of these nodes at separated places. Comparing the two upper snapshots in any of the vertical sequences, we see that an overall collapse-like event acts in between onto a structured net of cells as a contractive deformation. It somehow erases the cell structure, joining together clumps mainly along filaments, and therefore only very low relative angular momentum is involved at these $z \mathrm{~s}$. The collapse-like event shrinks the volume visualized at $z=3.5$ into that at $z=1.75$, where a central mass concentration fed

\footnotetext{
6 Indeed, as Figure 3 shows, the first snapshot at $t / t_{U}=0.13$ gives us the configuration of the bulge-to-be stars at the very beginning of the collapse-like event; the second one at $t / t_{U}=0.28$ roughly corresponds at the end of the fast phase; the third one at $t / t_{U}=0.38$ is just before the beginning of the major merger causing the strong stellar burst in the slow phase, and finally the fourth one at $t / t_{U}=0.59$ corresponds to the end of this burst.

7 Caustics are the elements of the cellular structure where dense mass elements show up at high zs. They are classified into walls, filaments, and nodes, and, at a given scale, these represent a temporal sequence of mass piling up. See Shandarin \& Zeldovich (1989) and Domínguez-Tenreiro et al. (2011) for more details.
} 

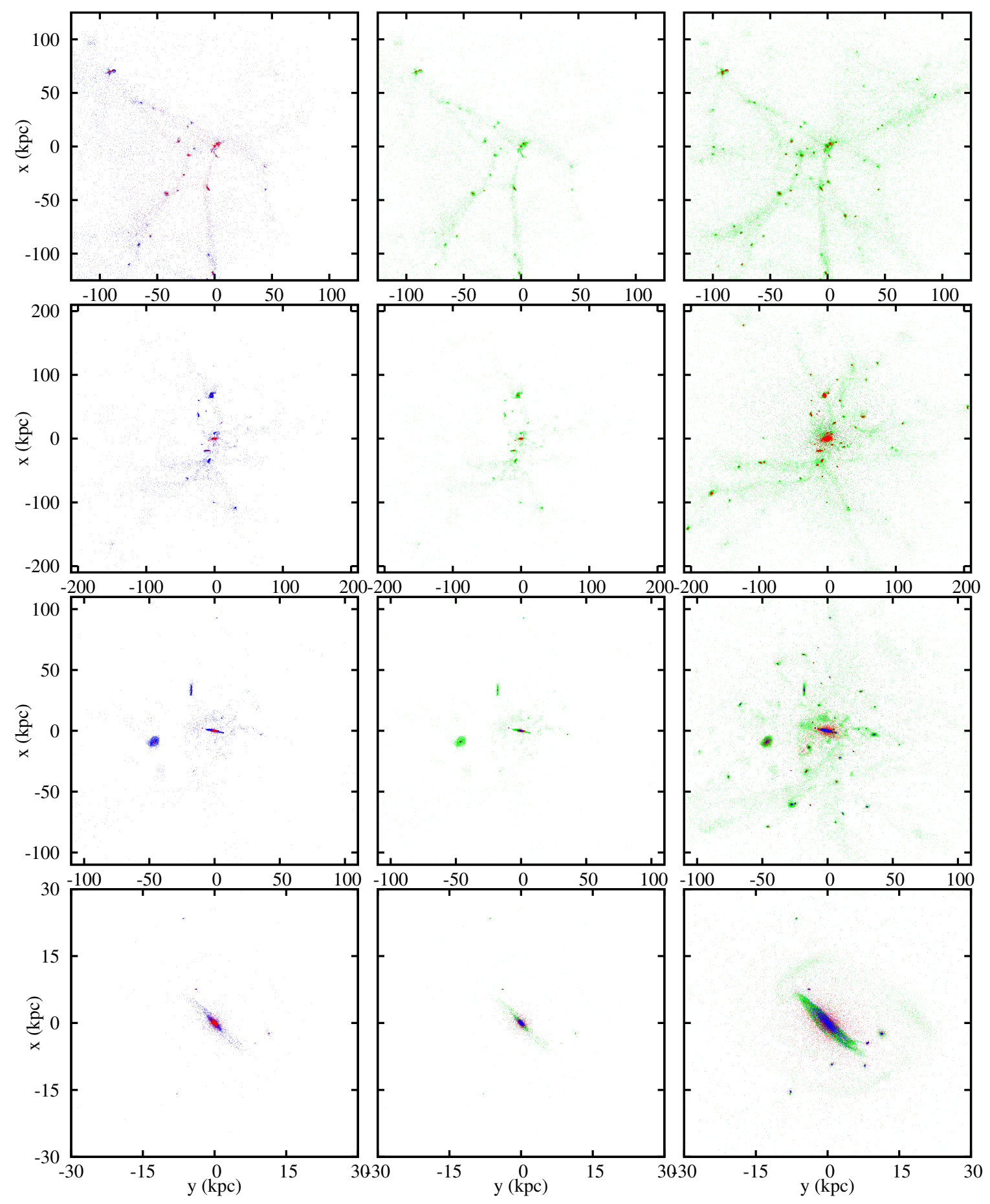

Figure 10. Different components of the bulge HD-5004A (left and central panels) and its surroundings (right panels) at redshifts $3.50,1.75,1.21$, and $0.58\left(t / t_{U}\right.$ of $0.13,0.28,0.38$, and 0.59 ), from top to bottom. The left panels give the positions of the bulge stellar particles identified at $z=0$ when traced back to each of the four $z$ s. Red corresponds to baryon particles (either gas or stars) whose transformation into stars occurs along the fast phase of bulge formation (i.e., at $t_{\text {form }} / t_{U}<0.31$, or equivalently, $z>1.54$ in this particular case), and blue to bulge baryon particles that become stars at later times. The same traced back positions are given in the central panels, but in this case with a color code representing particles properties at the zs plotted, namely green for cold gaseous particles $\left(T<10^{6} \mathrm{~K}\right)$, black for the hot ones, and red and blue for the stellar particles according to their time of birth (older and respectively younger than $z=1.54$ ). The right panels show the positions of all baryonic particles within a limiting radius of the galaxy's progenitor center using the same colors as in the central graphs.

by filaments stands out. Gas keeps on flowing through filaments toward the central regions between snapshots two and three. A diffuse component (i.e., outside caustics) is also evident at high $z$. This diffuse component tends to flow into caustics, therefore vanishing as evolution proceeds. Otherwise, by $z=1.21$ the filaments have practically been removed in favor of clumps and some cold gas in irregular structures. Moreover, two small gaseous disk-like structures and a third central disk with a stellar component, seen edge-on, have formed in the interval between the second and third snapshots. At $z=1.21$, the stars are at the center of these disks or at the center of other smaller gaseous structures. The young-to-be bulge stars begin to show up (blue points). Finally, after a major merger occurring between the third and fourth snapshots, by $z=0.58$ practically all the baryonic particles that are to form the HD-5004A bulge stars at $z=0$ come to be bound in a unique system (except for some small 
clumps). Most of the particles that have transformed into stars (red and blue at the central sequence) are at the center, while those that are still gaseous (in green) show up in a rebuilt disklike configuration. This configuration disappears later on and by $z=0.35$ (not shown in Figure 10) practically all the bulge particles are at their place within a sphere of radius $r_{\text {bulge }}$.

It is very useful to compare these evolutionary processes with the mass distribution of their surroundings visualized in the right graphs of Figure 10. This comparison makes it even clearer that bulge material has been involved in caustic formation at high $z$ although part of it remains as diffuse gas quite a while. Interestingly, a fraction of it has formed transient gaseous disks or has been a part of the former disk of the HD-5004A object.

We now analyze the differences in the assembly patterns of the young and old bulge stellar populations relative to the cosmic-web dynamics (left graphs in Figure 10). Most mass elements identified at $z=0$ as old stellar particles (red points) are already concentrated in caustics by $z=3.5$. These are the densest regions at this time. In contrast, an important fraction of the mass elements identified at $z=0$ as young stellar particles (blue points) are not yet involved in caustics. The difference increases as evolution proceeds. In fact, the strong contractive deformation acting between the first and second snapshots involves preferentially the old-stellar-to-be particles, in such a way that by $z=1.75$ the old component has a very small volume, while the young component still spans a scale of $\sim 200 \mathrm{kpc}$ and has an important diffuse gas fraction (with some nodes formed at local contractions as well). In the third snapshot, we see that a fraction of the mass elements that are to form young stars now shows disk-like patterns, while old stars are at the centers of these small disks. These disk-like configurations provide a key to explain the different kinematic properties of young bulge populations as compared to old ones, because of the angular momentum content of the former. As said above, the disks seen in the third snapshots merge. This causes their central old stars to form a unique bulge-like structure. At the same time, new stars-belonging to the young bulge-form and appear within it. On its turn, the young component, partially still as diffuse gas, forms structures (i.e., a disk in the case of HD-5004A as it can be seen in Figure 10) around the old stars spheroid. Some of the small nodes remain as satellites, carrying a small bit of old population at their centers. Finally, by $z=0.35$ (not shown in Figure 10), both the old and young bulge populations (or the gaseous particles to form the latter) are bound to their respective central spheroids, where they remain until $z=0$.

We draw attention to similarities with massive ellipticals (Domínguez-Tenreiro et al. 2011). In this case, stellar particles at $z=0$ exhibit a gaseous web-like morphology at $z \sim 3.5-6$, with scales of $\sim 1$ physical Mpc. The densest mass elements of this gaseous web, dynamically organized as attraction basins for mass flows, have already turned into stars by $z \sim 6$. At high $z$ these basins undergo fast contractive deformations which violently shrinks them in quasi-radial directions, therefore involving very low angular momentum. They can be described as collapse events with very complex geometries, causing high rates of dissipation and stellar formation out of the available gas, most of it being transformed into stars at the end of this process. Afterward, during the second phase, the mass assembly rate is much lower and is characterized by mergers involving significantly larger amounts of angular momentum.

To sum up, the old and young populations of the bulge HD$5004 \mathrm{~A}$ are not only segregated in their $z=0$ properties, but also show quite different assembly patterns in terms of the cosmicweb dynamics. Overall, this scenario also holds for the other four bulges. Indeed, most of the old stars formed at disjoint places within attraction basins during the early fast processes, and undergo an important assembly episode later on, through multiclump collapse-like events shrinking these basins in quasiradial directions. This assembly phase results in a relative low angular momentum of the merging clumps.

The ancestors of young bulge stars, on their turn, in many cases have been part of disk structures before arriving to the galaxy central regions. Different destabilizing processes can be responsible for this inward material transport, such as mergers or secular processes in the disks. Young bulge stars keep partial dynamical memory of their angular momentum content. These differences in the assembly patterns relative to the angular momentum involved would be a key piece in explaining the segregated properties of the two bulge components. Although our sample is small, the mass weight of the old component varies between $31 \%$ and $50 \%$. An even more important variation of this weight, together with the different inward mass transport mechanisms in the second assembly phase, could explain the important dispersion in bulge properties.

\section{SUMMARY, DISCUSSION, AND CONCLUSIONS}

We have analyzed the bulges of an inhomogeneous suite of five spiral galaxies emerging from high-resolution hydrodynamical simulations in a cosmological context. Our aim is to decipher the underlying physical processes in bulge assembly that could be responsible for their observed properties, focusing on relevant structural, kinematical, and chemical properties of their stellar populations and paying particular attention to their observationally suggested segregation by age.

Concerning the problem of bulge formation, the main particularity of this work is that we analyze simulated disk galaxies that are run with different codes (including different sub-grid physical prescriptions) where the chemical evolution has been carefully implemented, by using a common pipeline to measure the relevant stellar population properties. The simulations have been run with P-DEVA and GASOLINE. Feedback from massive stars is implemented explicitly in the two GASOLINE simulations through the blast-wave formulation (Stinson et al. 2006), while in P-DEVA simulations the effects of discrete energy injection are assumed to be on subgrid scales, resulting in the low star formation efficiency which is assumed to mimic them (see discussion in Agertz et al. 2011).

Although the details differ slightly, both P-DEVA and GASOLINE simulations trace the production and enrichment of chemical elements in broadly the same way. The newly formed elements, as by-products of stellar evolution and death, are released to the surrounding interstellar medium as increments in the metal content of nearby gas particles. The metals are diffused among gas particles, allowing the elements to be mixed and therefore mimicking the turbulent interstellar medium. Both codes consider the evolution of 11 elements. As we are looking for broad trends here, the relatively small differences in the implementation chemistry between the codes is not critical.

In this paper, we have studied in detail the mass-averaged three-dimensional sizes, shapes and kinematics, as well as stellar ages and element compositions of these five bulges. This is an intrinsic approach as opposed to looking for quantities closer to observations (i.e., light-averaged). We remain at this level because our aim in this paper is to discover the patterns of bulge mass assembly by focusing on their stellar population properties. 
We have found a satisfactory qualitative agreement with the latest observational data for the Milky Way as well as for other external bulges (see Section 1). Our results indicate that bulges in our sample have an old stellar population, formed at high $z$ at disjoint places within attraction basins, and joined together through their rapid quasi-radial multiclump collapse, where the relative angular momentum of the collapsing/merging clumps is low. A second phase follows, with lower mass assembly and SFRs, but with higher angular momenta. This phase shows a variety of assembly patterns: (1) only minor mergers without further significant SF bursts but an SF tail, (2) major mergers with secondary SF bursts, as the galaxy HD-5004A shows in Figures 3 and 10, and/or (3) secular evolution of the galactic disk, as is the case for G-1578411, which was shown in an earlier study to form its young bulge through secular processes after $z=1$, at least partially driven by a bar (Brook et al. 2012b).

The sizes, shapes, kinematics, stellar ages, and metal contents of the stellar populations formed in these distinct phases, can be nicely distinguished in simulated bulges. Indeed, we have found that the young component tends to occupy a smaller volume, to have disk-like morphology (note that G-1578411 is rather triaxial), to be more rotationally supported, to have roughly solar metallicities and sub-solar $\alpha$-element enhancements. The old population, by contrast, is more spheroid-like, has sub-solar metallicities and larger $\alpha$-element enhancements. On the other hand, no clear trend with age shows up in the Sérsic indices, a result in agreement with observations. The stellar metal content as well as the $[\mathrm{Mg} / \mathrm{Fe}]$ ratios of these bulges have segregated distributions and, in some cases, show two clearly distinguishably peaks corresponding to the old and young populations. These stellar populations are also clearly segregated by their loci in the $[\mathrm{Mg} / \mathrm{Fe}]$ versus $[\mathrm{Fe} / \mathrm{H}]$ plots, where the old population has only a mild slope, while the slope of the young population appears steeper, with the two populations meeting at the knee. This kind of behavior is expected given the different nucleosynthetic origin of $\alpha$ and Fe-group elements.

These trends have been shown to be robust against the different codes used, which have differences in their gravitational and hydrodynamical force integrators, in mass and spatial resolutions, simulation box sizes, star formation parameterizations, chemical feedback and evolution, and energetic feedback implementations. Also, by changing the bulge identification criteria from a simple radial cut to a kinematic-based one did not affect the age tendencies described above. The temporal cutoffs used to separate the two stellar populations have been chosen based on the shapes of the galaxy MATs and on the behavior of the SFRs at the bulge scale. Varying slightly these cutoffs does not affect these tendencies, either.

If we associate the old populations formed during the rapid phase with classical bulges, and the young ones formed during the slow phase with pseudo bulges, all the simulations in this paper show both classical and pseudo bulge components, but with varying relative masses. Therefore, the classical versus pseudo-bulge characteristics would be a question of degree, rather than nature. Otherwise, in all cases, measuring the light will result in higher relative contributions of the slow phase of bulge formation, i.e., pseudo bulges. In this respect, we note that the feedback in G-1536 is particularly effective at quenching star formation in the early phase, resulting in a relatively less significant (in mass) classical bulge population (see Table 2). This may be pointing toward the solution to the issue raised in Kormendy et al. (2010), who point out that a significant number of local, massive spiral galaxies have pseudo bulges rather than classical bulges.

These assembly patterns are reminiscent of the two phases found in hydrodynamical simulations by Domínguez-Tenreiro et al. (2006, 2011) and Oser et al. (2010) for more massive earlytype galaxies. The main difference lies in the percentage of gas transformed into stars at early epochs. In the case of massive ellipticals most of the available gas at the attraction basins for mass flows is transformed into stars during its "collapse" along the first phase. On the contrary, no such exhaustive gas consumption occurs for the less massive galaxies, where gas remains available along the slow phase, first as a diffuse component, and later on being part of different structures (the host galaxy disk itself, other small disk-like satellites and/or small clumps) before it is incorporated to the bulge.

If indeed real bulges follow a similar formation pattern as simulated ones, their observational features discussed in Section 1 can be nicely explained. More so, this approach provides a possible explanation for some apparently paradoxical observational results. For example, metal gradients could result from the different space distributions of the old and young populations, with the latter being more concentrated at the center due to dissipation. Also, bulge rejuvenation can be easily explained within this scenario.

We conclude that bulges can follow different assembly patterns, which can be summarized as two-phase processes (as in ellipticals) where non-exhaustive gas transformation into stars occurs in the fast phase (unlike in ellipticals), with the additional effects, along the slow phase, of major and minor mergers, as well as of disk secular instabilities, in some cases. These different patterns and their combinations in different epochs as well as in different proportions might explain the important dispersion in bulge properties observationally found.

The assembly of bulges is driven to a large extent by dynamical processes at larger scales. This is particularly true concerning bulge-forming starbursts. As our simulations show, the bulge mass aggregation is a delayed consequence of its host galaxy mergers in the slow phase, and the result of collapselike events in the fast one. We have shown that galaxy mass and feedback can affect the relative contributions of these two phases, although it is certain that specific basin deformation/ collapse processes, as well as merger histories will also play a role. A set of statistical samples of simulation runs with various physical parameters is needed in order to break the degeneracy with the merging history and provide further insights into the importance of the two phases of bulge formation.

We thank J. Oñorbe for sharing with us his analysis software and Patricia Sánchez-Blázquez for useful discussions. We also thank the anonymous referee for constructive comments. This work was partially supported by the MICINN (Spain) through the grants AYA2009-12792-C03-02 and AYA2009-12792-C0303 from the PNAyA, as well as by the regional Madrid V PRICIT program through the ASTROMADRID network (CAM S2009/ESP-1496) and the "Supercomputación y e-Ciencia" Consolider-Ingenio CSD2007-0050 project. We also thank the computer resources provided by BSC/RES (Spain) and the Centro de Computación Cientifíca (UAM, Spain). A. Obreja is supported by an FPI fellowship from MINECO, Spain, through the PNAyA.

\section{REFERENCES}

Agertz, O., Teyssier, R., \& Moore, B. 2011, MNRAS, 410, 1391 Athanassoula, E. 2005, MNRAS, 358, 1477 
Babusiaux, C., Gómez, A., Hill, V., et al. 2010, A\&A, 519, A77

Barbuy, B., Ortolani, S., Bica, E., \& Desidera, S. 1999, A\&A, 348, 783

Bensby, T., Adén, D., Meléndez, J., et al. 2011, A\&A, 533, A134

Binney, J. 1978, MNRAS, 183, 501

Binney, J., \& Tremaine, S. 1987, Galactic Dynamics (Princeton, NJ: Princeton Univ. Press)

Brook, C. B., Stinson, G., Gibson, B. K., Wadsley, J., \& Quinn, T. 2012a, MNRAS, 421, 3222

Brook, C. B., Stinson, G., Gibson, B. K., et al. 2012b, MNRAS, 419, 771

Bryan, G. L., \& Norman, M. L. 1998, ApJ, 495, 80

Bureau, M. 2002, in ASP Conf. Ser. 275, Disks of Galaxies: Kinematics, Dynamics and Peturbations, ed. E. Athanassoula, A. Bosma, \& R. Mujica (San Francisco, CA: ASP), 71

Calura, F., Gibson, B. K., Michel-Dansac, L., et al. 2012, MNRAS, 427, 1401

Carollo, C. M., Scarlata, C., Stiavelli, M., Wyse, R. F. G., \& Mayer, L. 2007, ApJ, 658,960

Carollo, C. M., Stiavelli, M., de Zeeuw, P. T., Seigar, M., \& Dejonghe, H. 2001, ApJ, 546, 216

Chabrier, G. 2003, ApJ, 586, L133

Clarkson, W., Sahu, K., Anderson, J., et al. 2008, ApJ, 684, 1110

Combes, F. 2009, in ASP Conf. Ser. 419, Galaxy Evolution: Emerging Insights and Future Challenges, ed. S. Jogee, I. Marinova, L. Hao, \& G. A. Blanc (San Francisco, CA: ASP), 31

Cook, M., Lapi, A., \& Granato, G. L. 2009, MNRAS, 397, 534

De Propris, R., Rich, R. M., Kunder, A., et al. 2011, ApJ, 732, L36

de Zeeuw, T., \& Franx, M. 1991, ARA\&A, 29, 239

Debattista, V. P., Carollo, C. M., Mayer, L., \& Moore, B. 2004, ApJ, 604, L93

Doménech-Moral, M., Martínez-Serrano, F. J., Domínguez-Tenreiro, R., \& Serna, A. 2012, MNRAS, 421, 2510

Domínguez-Tenreiro, R., Oñorbe, J., Martínez-Serrano, F., \& Serna, A. 2011, MNRAS, 413, 3022

Domínguez-Tenreiro, R., Oñorbe, J., Sáiz, A., Artal, H., \& Serna, A. 2006, ApJ, 636, L77

Eliche-Moral, M. C., González-García, A. C., Balcells, M., et al. 2011, A\&A, 533, 104

Ellis, R. S., Abraham, R. G., \& Dickinson, M. 2001, ApJ, 551, 111

Erwin, P. 2008, in IAU Symp. 245, ed. M. Bureau, E. Athanassoula, \& B. Barbuy (Cambridge: Cambridge Univ. Press), 113

Feltzing, S., \& Gilmore, G. 2000, A\&A, 355, 949

Ferreras, I., Wyse, R. F. G., \& Silk, J. 2003, MNRAS, 345, 1381

Fisher, D. B., \& Drory, N. 2008, AJ, 136, 773

Fisher, D. B., \& Drory, N. 2010, APJ, 716, 942

Franx, M. 1993, in IAU Symp. 153, Galactic Bulges, ed. H. Dejonghe \& H. J. Habing (Cambridge: Cambridge Univ. Press), 243

Freeman, K. C. 1970, ApJ, 160, 811

Freyer, T., Hensler, G., \& Yorke, H. W. 2006, ApJ, 638, 262

Fulbright, J. P., McWilliam, A., \& Rich, R. M. 2007, ApJ, 661, 1152

Gonzalez, O. A., Rejkuba, M., Zoccali, M., et al. 2011, A\&A, 530, A54

González-García, A. C., \& van Albada, T. S. 2005, MNRAS, 361, 1030

Governato, F., Brook, C. B., Brooks, A. M., et al. 2009, MNRAS, 398, 312

Grevesse, N., \& Sauval, A. J. 1998, SSRv, 85, 161

Graham, A.W. 2011, in Planets, Stars, and Stellar Systems, arXiv:1108.0997

Groenewegen, M. A. T., \& Blommaert, J. A. D. L. 2005, A\&A, 443, 143

Guedes, J., Callegari, S., Madau, P., \& Mayer, L. 2011, ApJ, 742, 76

Hill, V., Lecureur, A., Gomez, A., et al. 2011, A\&A, 534, 80

Hopkins, P. F., Bundy, K., Croton, D., et al. 2010, ApJ, 715, 202

Iwamoto, K., Brachwitz, F., Nomoto, K., et al. 1999, ApJS, 125, 439

Jablonka, P., Gorgas, J., \& Goudfrooij, P. 2007, A\&A, 474, 763

Johnson, C. I., Rich, R. M., Fulbright, J. P., Valenti, E., \& McWilliam, A. 2011, ApJ, 732, 108

Johnson, C. I., Rich, R. M., Kobayashi, C., \& Fulbright, J. P. 2012, ApJ, 749, 175

Katz, N. 1992, ApJ, 391, 502

Kauffmann, G., Colberg, J. M., Diaferio, A., \& White, S. D. M. 1999, MNRAS, 303, 188

Kawata, D., \& Gibson, B. K. 2003, MNRAS, 340, 908
Kay, S. T., Pearce, F. R., Frenk, C. S., \& Jenkins, A. 2002, MNRAS, 330,113

Kobayashi, C., \& Nakasato, N. 2011, ApJ, 729, 16

Kobayashi, C., Tsujimoto, T., \& Nomoto, K. 2000, ApJ, 539, 26

Kormendy, J. 1982, in Saas-Fee Advanced Course 12: Morphology and Dynamics of Galaxies, ed. L. Martinet \& M. Mayor (Sauverny, Switzerland: Observatoire de Geneve), 113

Kormendy, J., Drory, N., Bender, R., \& Cornell, M. E. 2010, ApJ, 723, 54

Kormendy, J., \& Kennicutt, R. C., Jr. 2004, ARA\&A, 42, 603

Kroupa, P., Tout, C. A., \& Gilmore, G. 1993, MNRAS, 262, 545

Lecureur, A., Hill, V., Zoccali, M., et al. 2007, A\&A, 465, 799

MacArthur, L. A., González, J. J., \& Courteau, S. 2009, MNRAS, 395, 28

Martínez-Serrano, F. J., Serna, A., Domínguez-Tenreiro, R., \& Mollá, M. 2008, MNRAS, 388, 39

Matteucci, F., \& Brocato, E. 1990, ApJ, 365, 539

McDonald, M., Courteau, S., Tully, R. B., \& Roediger, J. 2011, MNRAS, 414, 2055

McWilliam, A., \& Rich, R. M. 1994, ApJS, 91, 749

Mollá, M., Ferrini, F., \& Gozzi, G. 2000, MNRAS, 316, 345

Monaghan, J. J. 2005, RPPh, 68, 1703

Moorthy, B. K., \& Holtzman, J. A. 2006, MNRAS, 371, 583

Morelli, L., Corsini, E. M., Pizzella, A., et al. 2012, MNRAS, 423, 962

Nakasato, N., \& Nomoto, K. 2003, ApJ, 588, 842

Ness, M., \& Freeman, K. 2012, Eur. Phys. J. Web Conf., 19, 6003

Ness, M., Freeman, K., Athanassoula, E., et al. 2012, ApJ, 756, 22

Nomoto, K., Hashimoto, M., Tsujimoto, T., et al. 1997, NuPhA, 616, 79

Okamoto, T. 2012, arXiv:1203.5372

Ortolani, S., Renzini, A., Gilmozzi, R., et al. 1995, Natur, 377, 701

Oser, L., Ostriker, J. P., Naab, T., Johansson, P. H., \& Burkert, A. 2010, ApJ, 725,2312

Peletier, R. F. 2008, in ASP Conf. Ser. 390, Pathways Through an Eclectic Universe, ed. J. H. Knapen, T. J. Mahoney, \& A. Vazdekis (San Francisco, CA: ASP), 232

Peletier, R. F., Balcells, M., Davies, R. L., et al. 1999, MNRAS, 310, 703

Peletier, R. F., Falcón-Barroso, J., Bacon, R., et al. 2007, MNRAS, 379, 445

Prugniel, P., Maubon, G., \& Simien, F. 2001, A\&A, 366, 68

Rahimi, A., Kawata, D., Brook, C. B., \& Gibson, B. K. 2010, MNRAS, 401, 1826

Robin, A. C., Marshall, D. J., Schultheis, M., \& Reylé, C. 2012, A\&A, 538, A106

Salpeter, E. E. 1955, ApJ, 121, 161

Samland, M., \& Gerhard, O. E. 2003, A\&A, 399, 961

Sánchez-Blázquez, P., Ocvirk, P., Gibson, B. K., Pérez, I., \& Peletier, R. F. 2011, MNRAS, 415, 709

Serna, A., Domínguez-Tenreiro, R., \& Sáiz, A. 2003, ApJ, 597, 878

Shandarin, S. F., \& Zeldovich, Y. B. 1989, RvMP, 61, 185

Shen, S., Wadsley, J., \& Stinson, G. 2010, MNRAS, 407, 1581

Soto, M., Kuijken, K., \& Rich, R. M. 2012, A\&A, 540, 48

Soto, M., Rich, R. M., \& Kuijken, K. 2007, ApJ, 665, L31

Stinson, G., Brook, C., Macciò, A.V., et al. 2012, MNRAS, 428, 129

Stinson, G. S., Bailin, J., Couchman, H., et al. 2010, MNRAS, 408, 812

Stinson, G. S., Seth, A., Katz, N., et al. 2006, MNRAS, 373, 1074

Talbot, R. J., Jr., \& Arnett, W. D. 1973, ApJ, 186, 51

Thomas, D., \& Davies, R. L. 2006, MNRAS, 366, 510

Tissera, P. B., \& Dominguez-Tenreiro, R. 1998, MNRAS, 297, 177

Tsujimoto, T., \& Bekki, K. 2012, ApJ, 747, 125

van Loon, J. T., Gilmore, G. F., Omont, A., et al. 2003, MNRAS, 338, 857

Wechsler, R. H., Bullock, J. S., Primack, J. R., Kravtsov, A. V., \& Dekel, A. 2002, ApJ, 568, 52

Weisberg, S. 2002, J. Stat. Soft., 7, 23

Woosley, S. E., \& Weaver, T. A. 1995, ApJS, 101, 181

Wyse, R. F. G. 1999, Ap\&SS, 267, 145

Zhao, D. H., Mo, H. J., Jing, Y. P., \& Börner, G. 2003, MNRAS, 339, 12

Zhao, H., Spergel, D. N., \& Rich, R. M. 1994, AJ, 108, 2154

Zoccali, M., Hill, V., Lecureur, A., et al. 2008, A\&A, 486, 177

Zoccali, M., Lecureur, A., Barbuy, B., et al. 2006, A\&A, 457, L1 